\title{
Imperfections of the North-Atlantic wind-driven ocean circulation: Continental geometry and Windstress shape
}

\author{
Henk A. Dijkstra and M. Jeroen Molemaker \\ Institute for Marine and Atmospheric Research Utrecht \\ Utrecht University \\ Utrecht, the Netherlands \\ Revised for J. Marine Research
}

Version of September 1, 1998

Corresponding Author:

Henk A. Dijkstra

Institute for Marine and Atmospheric research Utrecht

Department of Physics and Astronomy

Utrecht University

Princetonplein 5, 3584 CC Utrecht

The Netherlands

Phone: -31-30-2533276; Fax: -31-30-2543163

Email: dijkstra@fys.ruu.nl 


\begin{abstract}
Multiple equilibria of the wind-driven gyres have been found in idealized quasigeostrophic and shallow water models. In this paper we demonstrate that multiple equilibria persist within a reduced gravity shallow water model under quite realistic continental geometry and windstress forcing for the North-Atlantic. Multiple mean flow patterns of the Gulf Stream exist and differ with respect to their separation behavior along the North-American coast. The origin of these equilibria is investigated by determining the structure of steady solutions within a hierarchy of equivalent barotropic ocean models using continuation techniques. Within each model, the magnitude of lateral friction is used as a control parameter. It is shown that symmetry breaking, found in a quasi-geostrophic model for a rectangular ocean basin with idealized wind forcing is at the origin of two different mean states of the Gulf Stream. The steady states found become unstable only to a small number of oscillatory modes, which either have intermonthly or interannual periods. The modes of variability remain strongly related through the hierarchy of models indicating that their physics is not strongly dependent on the shape of the continents but is controlled by internal ocean dynamics.
\end{abstract}




\section{Introduction}

Many concepts of geophysical fluid dynamics and physical oceanography have been developed from results of theoretical models dealing with highly idealized situations. For example, steady linear quasi-geostrophic rectangular basin models have lead to the explanation of the intensification of western boundary currents in the ocean (Stommel, 1948; Munk, 1950). Nonlinearities due to advection of vorticity introduce north-south asymmetries and are responsible for strong recirculation regions. In the fully inertially dominated regime, special types of motion may occur, i.e. Fofonoff modes (Fofonoff, 1954) or modon type solutions (Stern, 1975). In this regime, several types of sensitivities arise to the boundary conditions and the parameterization of frictional processes. (Pedlosky, 1996).

In reality, these flows are unsteady, one of the reasons being that they are susceptible to several kinds of instabilities. Advances in the understanding of the instability mechanisms, e.g. barotropic and baroclinic instability, has been obtained through their study in idealized models (Pedlosky, 1987), for example flows in $\beta$-plane channels. The growth of perturbations and their interaction with the background state and with each other leads to a rectification of the mean state and synoptic scale time-dependent features, generally referred to as oceanic eddies. The physics of these phenomena can be studied in detail in the weakly nonlinear regime, for which only a small band of unstable wavenumbers is involved (Pedlosky, 1987; Van der Vaart and Dijkstra, 1997).

Recently, the stability of full basin flows have been addressed and multiple equilibria of the double gyre wind-driven circulation in rectangular basins have been found in quasi-geostrophic models (Cessi and Ierley, 1995; Dijkstra and Katsman, 1997). Under symmetric (with respect to the mid-basin axis) windstress forcing, it is found that if lateral friction is small enough, the anti-symmetric double gyre solution destabilizes and asymmetric stable steady states exist. The mechanism of the existence of the 
asymmetric double gyre flows is related to a symmetry breaking pitchfork bifurcation and has a barotropic origin. Also in reduced gravity shallow water models, multiple equilibria exist in rectangular basins (Speich et al., 1995). In both type of models, the asymmetric flows destabilize through Hopf bifurcations as the friction is further decreased, leading to time-dependent behavior. There are strong indications that in these idealized models, the time-dependent behavior is controlled by only a few degrees of freedom in the strongly nonlinear regime (Berloff and Meacham, 1997; Meacham and Berloff, 1998).

Over the last decades, eddy resolving ocean models have been developed which are able to produce many features observed in reality, e.g. the formation and propagation of meso-scale eddies (McWilliams, 1996). In quite idealized situations, many studies have addressed the problem of eddy-mean flow interaction (Holland, 1978). An accurate representation of the eddy field turns out to be crucial to obtain the correct properties of the large scale flow (Holland and Schmitz, 1985). The eddies are also essential to understand the temporal variability of the flow, which occurs on time scales from several weeks to several years (Schmitz and Holland, 1982). Although much of the observed variability may be forced by atmospheric noise, results from eddy-resolving models indicate that intrinsic variability of the ocean is large and not only in the region of strong currents but over the whole basin (Miller et al., 1987).

Ocean general circulation models (OGCM's), having a realistic continental geometry and bathymetry and incorporating the thermohaline circulation, are run now with an eddy-resolving resolution, not only for the Atlantic (New et al., 1996) but also on a global scale (Semtner and Chervin, 1992). These results also show large internal variability on a wide range of time scales and the influence of the eddies on the mean flow. The interpretation of results of these models is not without problems, because of the multitude of physical effects which can influence a phenomenon under study. For example, it appears that several physical processes control the mean flow path of the 
Gulf Stream, in particular its separation near Cape Hatteras. In models of $1^{\circ}$ horizontal resolution (Holland and Bryan, 1994), separation of the Gulf Stream is very diffuse between Cape Hatteras and Newfoundland and no recirculation regions are present. For very high resolution models of $\frac{1}{6}^{\circ}$ (Beckmann et al., 1994; Bryan et al., 1995) the time mean state shows a large anti-cyclonic gyre north of Cape Hatteras giving an actual separation north of the observed position with undesirable consequences for the simulated heat transport.

The problem of Gulf Stream separation has been recently reviewed in Dengg et al. (1996). External factors such as bottom topography, continental geometry and the structure of the windstress curl each have been identified to be important factors. Also several mechanisms which are related to internal ocean dynamics have been suggested, such as outcropping of isopycnals (Gangopadhyay et al., 1992; Chassignet and Bleck, 1993), vorticity crisis (Cessi et al., 1995) and inertial overshoot (Dengg, 1993). Combined factors such as JEBAR may also be important (Meyers et al., 1996). Several studies have been performed to isolate only one or two factors but then the relation between these results and those in more realistic models is hard to establish. The same holds for the internal variability of the models. Many parameter studies which have been performed with idealized models cannot easily be done with the OGCM's.

In intermediate models of the Gulf Stream region, with less idealized continents, signatures of multiple flow states have been found, although not for exactly the same conditions (Dengg, 1993). Combined with the fact that multiple equilibria do exist for the double gyre flows mentioned earlier (Cessi and Ierley, 1995), this suggests that multiple equilibria may play a role in the problem of ocean models to simulate the correct Gulf Stream separation. If so, one first has to demonstrate that these exist in realistic geometry and under realistic windstress forcing. In this paper, we demonstrate the simultaneous existence of two mean flow paths of the Gulf Stream within a shallow water model. 
In order to determine the (topological) origin of these multiple equilibria, we use a hierarchy of models. At one end of the model hierarchy is a 1.5 layer quasi-geostrophic model in a rectangular basin and at the other end is a 1.5 shallow water model with realistic North-Atlantic continental geometry. We compute the structure of the equilibria within each model using continuation techniques. In this way, a connection is established - in so far as the influence of continental geometry and (equivalent) barotropic phenomena is concerned - between results of a simple model and the more complex model of the North-Atlantic wind-driven ocean circulation. The existence of the two possible mean flow paths of the Gulf Stream near Cape Hatteras can be traced back to originate from the symmetry breaking mechanism of the double gyre flow in the 1.5 layer quasi-geostrophic model. Modes of variability which arise as instabilities of

the steady states have either intermonthly or interannual period and are continuously related through the model hierarchy.

\section{Formulation}

As basically two different type of models are considered in this study, each with slightly different model parameters, the equations and their non-dimensionalization are presented below. Consider an ocean basin with an arbitrary horizontal domain $\Omega$ (with a typical horizontal lengthscale $L$ ) and bounded by a closed contour $\Gamma$, on a midlatitude $\beta$-plane with Coriolis parameter $f\left(f=f_{0}+\beta_{0} y\right)$. An active layer of mean depth $D$ with density $\rho$ is situated above a slightly heavier deeper layer having a density $\rho+\Delta \rho$ which is supposed to be motionless. The interface between the two layers, the thermocline, is able to deform and the reduced gravity $g^{\prime}$ is given by $g^{\prime}=g \Delta \rho / \rho$. The flow is driven by a windstress $\mathbf{T}(x, y)=\tau_{0}\left(\tau^{x}, \tau^{y}\right)$, where $\tau_{0}$ is the amplitude and $\left(\tau^{x}, \tau^{y}\right)$ provides the spatial pattern. Lateral friction, with lateral friction coefficient $A_{H}$ and bottom friction, with bottom friction coefficient $\epsilon_{0}$, are the dissipative mechanisms in 
both models.

\section{a. The 1.5 layer quasi-geostrophic (QG) model}

Characteristic horizontal and vertical length scales in this model are $L$ and $D$, a velocity-scale is the depth averaged velocity $U$, the advective time-scale is $L / U$ and $\tau_{0}$ is the characteristic amplitude of the windstress. If the Rossby number $\epsilon=U /\left(f_{0} L\right)$ is small, quasi-geostrophic theory is a useful approximation of the dynamics of the flow (Pedlosky, 1987). In this case, the dimensionless potential vorticity equation describing the flow becomes

$$
\begin{aligned}
{\left[\frac{\partial}{\partial t}+u \frac{\partial}{\partial x}+v \frac{\partial}{\partial y}\right][\zeta-F \psi+\beta y] } & =R e^{-1} \nabla^{2} \zeta+\alpha_{Q}\left[\frac{\partial \tau^{y}}{\partial x}-\frac{\partial \tau^{x}}{\partial y}\right]-r \zeta \\
\zeta & =\nabla^{2} \psi
\end{aligned}
$$

In the equations above, the streamfunction $\psi$ and vorticity $\zeta$ are used, with the zonal and meridional geostrophic velocity vector determined by $(u, v)=(-\partial \psi / \partial y, \partial \psi / \partial x)$. On the boundary $\Gamma$ of the domain no-slip conditions are prescribed, i.e.

$$
\mathbf{x} \varepsilon \Gamma: \quad \psi=0, \quad \nabla \psi \cdot \mathbf{n}=0
$$

where $\mathbf{n}$ is the outward normal on $\Gamma$.

Five parameters appear in the system of equations (1), i.e. the Reynolds number $R e$, the strength of the planetary vorticity gradient $\beta$, the strength of the windstress forcing $\alpha_{Q}$, the rotational Froude number $F$ and the bottom friction parameter $r$. These parameters are defined as

$$
R e=\frac{U L}{A_{H}} ; \beta=\frac{\beta_{0} L^{2}}{U} ; \alpha_{Q}=\frac{\tau_{0} L}{\rho D U^{2}} ; F=\frac{f_{0}^{2} L^{2}}{g^{\prime} D} ; r=\frac{\epsilon_{0} L}{U}
$$

The parameter $F=\left(\frac{L}{\lambda}\right)^{2}$ relates to the internal Rossby deformation radius through $\lambda=$

$\left(g^{\prime} D\right)^{\frac{1}{2}} / f_{0}$. Since $U$ is not an independent parameter, there are only four independent 
control parameters in the governing equations of the model. Additional parameters are introduced by the shape of the domain, for example, if a rectangular basin with length $L$ and width $B$ is considered, a new parameter is the aspect ratio of the domain. Standard values of the dimensional and dimensionless parameters are listed in Table 1. The Rossby deformation radius $\lambda$ for these parameter values is about $75 \mathrm{~km}$.

\section{b. The 1.5 layer shallow water (SW) model}

In a shallow water formulation, the Rossby number $\epsilon$ need not be small and, for example, deviations of the thermocline from its equilibrium value $D$ can be large. In the usual notation, the velocities in eastward and northward directions are indicated by $u$ and $v$, respectively and $h$ is the thickness of the upper layer (with equilibrium value $D)$. The governing shallow water equations are non-dimensionalized using scales $L, D$, $U^{*}, L / U^{*}$ and $\tau_{0}$ for length, layer depth, velocity, time and windstress, respectively, and become (Jiang et al., 1995; Speich et al., 1995)

$$
\begin{aligned}
\epsilon\left(\frac{\partial u}{\partial t}+\mathbf{u} \cdot \nabla u\right)-(1+\epsilon \beta y) v & =-\epsilon F^{*} \frac{\partial h}{\partial x}+E \nabla^{2} u-r u+\alpha_{S} \frac{\tau^{x}}{h} \\
\epsilon\left(\frac{\partial v}{\partial t}+\mathbf{u} \cdot \nabla v\right)+(1+\epsilon \beta y) u & =-\epsilon F^{*} \frac{\partial h}{\partial y}+E \nabla^{2} v-r v+\alpha_{S} \frac{\tau^{y}}{h} \\
\frac{\partial h}{\partial t} & =-\frac{\partial(h u)}{\partial x}-\frac{\partial(h v)}{\partial y}
\end{aligned}
$$

On the boundary $\Gamma$ of the domain no-slip conditions are prescribed, i.e.

$$
\mathbf{x} \varepsilon \Gamma: \quad u=v=0
$$

The parameters in these equations are the Rossby number $\epsilon$, the strength of the planetary vorticity gradient $\beta$, the Froude number $F^{*}$, the Ekman number $E$, the windstress coefficient $\alpha_{S}$ and the bottom friction parameter $r$. Expressions for these parameters are

$$
\epsilon=\frac{U^{*}}{f_{0} L} ; F^{*}=\frac{g^{\prime} D}{U^{* 2}} ; E=\frac{A_{H}}{f_{0} L^{2}} ; \alpha_{S}=\frac{\tau_{0}}{\rho f_{0} H U^{*}} ; \beta=\frac{\beta_{0} L^{2}}{U^{*}} ; r=\frac{\epsilon_{0}}{f_{0}}
$$


and since again $U^{*}$ is not independent, the governing equations contain five independent parameters. Standard values of the parameters in this model are listed in Table 2 and the internal Rossby deformation radius is the same as in the quasi-geostrophic model.

The shallow water model differs only in one detail from that used in Speich et al. (1995). The parameterization of lateral friction is different, i.e. in the zonal momentum balance the difference is reflected by $\nabla^{2}(h u)$ versus $h \nabla^{2} u$. The latter formulation, for example used in Holland and Lin (1975), occurs more consistently in deriving the shallow water model with the horizontal velocities assumed depth-independent. With this formulation it is not a priori guaranteed (i.e. not easily to proof) that the dissipation operator in both the steady state and linear stability problem is negative definite. However, it turns out for all results presented below (through a postiori calculation) that this is actually the case and hence there is no problem in using the present form of lateral momentum exchange.

\section{c. Numerical methods}

For the QG model, a finite difference discretization on an equidistant grid was used as described in Dijkstra and Katsman (1997). Within the SW model, a finite element discretization was used, with Taylor Hood triangular elements as described in Molemaker and Dijkstra (1998). In each case, a set of nonlinear algebraic equations of the form

$$
\mathbf{F}(\mathbf{u}, \mathbf{p})=0
$$

emerges from the discretization of the steady equations. Here $\mathbf{u}$ is a $d$-dimensional vector consisting of the unknowns at the gridpoints, $\mathbf{p}$ is the $p$-dimensional vector of parameters and $\mathbf{F}$ is a nonlinear mapping from $R^{d} \times R^{p} \rightarrow R^{d}$, where $d$ indicates the number of degrees of freedom. To determine branches of steady solutions of the equations $(7)$ as one of the parameters (say $\mu$ ) is varied, the pseudo-arclength method is used. The branches $(\mathbf{u}(s), \mu(s))$ are parametrized by an 'arclength' parameter $s$. An 
additional equation is obtained by 'normalizing' the tangent

$$
\dot{\mathbf{u}}_{0}^{T}\left(\mathbf{u}-\mathbf{u}_{0}\right)+\dot{\mu}_{0}\left(\mu-\mu_{0}\right)-\Delta s=0
$$

where $\left(\mathbf{u}_{0}, \mu_{0}\right)$ is an analytically known starting solution or a previously computed point on a particular branch and $\Delta s$ is the steplength. Solutions to these equations in parameter space are obtained using methods as described in Dijkstra et al. (1995).

To calculate a steady state solution of the system of equations (7) an extra condition for $h$ is required to regularize the equations, since $h$ is determined up to an additive constant. In a previous study (Speich et al., 1995) the layer depth at one point was kept fixed at a prescribed value. The correct condition to use is an integral condition for $h$ over the domain $\Omega$ that removes the ambiguity from the layer depths, i.e.

$$
\int_{\Omega} h d x d y=|\Omega|
$$

which is an expression of conservation of mass of the upper layer. The integral is equal to $|\Omega|$, the (dimensionless) area of the domain, since the layer depth is scaled with $D$. In models that integrate the equations in time, this regularization problem is absent, since the integral of the layer depth is set by the initial conditions.

When a steady state is determined, the linear stability of the solution is considered and transitions that mark qualitative changes such as transitions to multiple equilibria (pitchfork bifurcations of limit points) or periodic behavior (Hopf bifurcations) can be detected. The linear stability analysis amounts to solving a generalized eigenvalue problem of the form

$$
\mathcal{A x}=\sigma \mathcal{B} \mathbf{x}
$$

where $\mathcal{A}$ and $\mathcal{B}$ are non-symmetric matrices. Solution techniques for these problems are presented in Dijkstra et al. (1995) and bifurcations are detected from crossings of 
$\sigma$ with the imaginary axis (Guckenheimer and Holmes, 1983).

In the results below, the strategy is to 'deform' the steady solutions from simple rectangular geometry and idealized wind stress to the full complex geometry and realistic windstress field of the North-Atlantic basin.

\section{Results: Idealized case}

The domain is rectangular with the width $B$ of the basin being $2000 \mathrm{~km}$. This introduces the aspect ratio $A=B / L$ as a new parameter (with $A=2$ ) similar to that used in earlier studies (Speich et al., 1995). In this section, the windstress forcing on the domain $[0,1] \times[0, A]$ is idealized as

$$
\begin{aligned}
\tau_{S}^{x}(x, y) & =-\cos (2 \pi y / A) \\
\tau_{S}^{y}(x, y) & =0
\end{aligned}
$$

\section{a. Quasi-geostrophic model}

Calculations were performed on an equidistant $48 \times 96$ grid (a resolution of about $20 \mathrm{~km}$ ) which proved to give sufficiently accurate solutions. As in Dijkstra and Katsman (1997), we use Re as a control parameter. The bifurcation diagram for the standard values of parameters is shown in Fig. 1, as a plot of $R e$ against $\Psi$, the latter being the sum of maximum and minimum value of the streamfunction over the field. The quantity $\Psi$ is zero when the streamfunction solution is anti-symmetric. Drawn (dotted) branches indicate stable (unstable) steady states, whereas bifurcation points are indicated by markers. The anti-symmetric double gyre solution (similar to Figure 2(a) in Dijkstra and Katsman (1997)) is stable at small Re. It becomes unstable through a stationary instability at $R e=52$ (the point $P$ in Fig. 1) and asymmetric solutions stabilize. One of these solutions is shown for $R e=67$ in Fig. 2 as a contour plot of the streamfunction. 
In the figure, the contour levels are scaled with respect to the maximum value of the field. The asymmetric solution in Fig. 2 has its jet displaced downward. The solution on the other asymmetric branch at the same value of Re is symmetrically related to that in Fig. 2 and has the jet displaced upwards.

Both asymmetric solutions destabilize through a Hopf bifurcation ( $H$ in Fig. 1$)$ at $R e=64$. The pattern of the mode which is destabilized is determined from the eigenvector $\mathbf{x}=\mathbf{x}_{r}+i \mathbf{x}_{i}$ associated with the eigenvalue $\sigma=\sigma_{r}+i \sigma_{i}$ in (10). These span an oscillatory mode given by

$$
\mathbf{\Phi}(t)=\cos \left(\sigma_{i} t\right) \mathbf{x}_{r}-\sin \left(\sigma_{i} t\right) \mathbf{x}_{i}
$$

with dimensional period $T=2 \pi L /\left(U \sigma_{i}\right)$ s.

The patterns of $\mathbf{x}_{r}$ and $\mathbf{x}_{i}$ of the slightly unstable eigenmode at $R e=67$ are plotted in Fig. 3, with the angular frequency $\sigma_{i}$ and growth rate $\sigma_{r}$ given in the caption. Note that the propagation features have to be determined by first looking at $\mathbf{x}_{i}=$ $\Phi\left(-\pi /\left(2 \sigma_{i}\right)\right)$ and than at $\mathbf{x}_{r}=\Phi(0)$, which is the pattern of the mode just a quarter period later. The unstable mode has an interannual period and it was argued in Dijkstra and Katsman (1997) that the period is controlled by the advective time scale of the gyre. The axis connecting the extrema of the perturbations, below referred to as the principal axis, is nearly orthogonal to the direction of the jet of the steady state (Fig. 2) and the perturbations propagate southwestward.

As in Dijkstra and Katsman (1997), the destabilization of the asymmetric solutions occurs through a small number of modes. The second mode (not shown) is still quite stable at $R e=67$, and its pattern could therefore not be accurately computed. However, the not yet fully converged results indicate that it has an intermonthly period, and its pattern is characterized by a principle axis which points in zonal direction, unrelated to the direction of the jet of the steady state. The perturbations propagate in zonal direction and have similar characteristics as the basin modes in Dijkstra and Katsman 
(1997). This second mode destabilizes at larger $R e$ through the increase in horizontal shear of the basic state. The third mode (also not shown) has a similar time scale as the second, but its (negative) growth factor is about twice that of the second mode and it is therefore very stable.

\section{b. Shallow water model}

The finite element grid generated for the rectangular basin of aspect ratio $A=2$ is shown in Figure 4 and consists of 2442 triangular elements that become smaller towards the western boundary. The resulting system of nonlinear algebraic equations has 11322 degrees of freedom. Using the finite element model and continuation software, branches of stationary solutions are computed using the Ekman number as control parameter (fixing all other parameters as in Table 2).

The basic bifurcation diagram (Figure 5(a)) consists of a perturbed pitchfork bifurcation (Golubitsky and Schaeffer, 1985). On the vertical axis, the maximum northward volume transport $\phi$ (in Sv) over a section is shown which is calculated as

$$
\phi=\left(U^{*} H L\right) \max \int_{0}^{x_{e}} v h d x
$$

where the maximum is taken both over $y$ and $x_{e}$. Again, drawn (dotted) branches represent stable (unstable) solutions. Compared to the result in the quasi-geostrophic model, the lower branch is no longer connected to the branch that originates from the higher Ekman number regime. On the upper branch in Fig. 5(a), a Hopf bifurcation occurs at $E=0.34 \times 10^{-5}$ and is marked with $H_{1}$. The steady state at this value of $E$ is shown as a contour plot of $h$ in Fig. 5(b) and shows a north-south asymmetry with the jet displaced southwards from the mid-axis of the basin. On the lower branch in Fig. 5(a), the interval of stable solutions is bounded by a limit point $(L)$ at $E=0.5210^{-5}$ and a Hopf bifurcation $\left(H_{2}\right)$ at $E=0.3610^{-5}$. The steady solution at the Hopf bifurcation $H_{2}$ (Fig. 5(c)) has a northward displacement of the jet and is nearly symmetrically related to that in Fig. 5(b) 
At the Hopf bifurcations, the steady state becomes unstable to one oscillatory mode. For the shallow water model, the spectral separation between the modes is much smaller than for the QG model and three modes become unstable nearly at the same conditions. The real and imaginary parts of the eigenvector of the first three modes are shown at the Hopf bifurcation $H_{1}$ in Fig. 6. The first mode (panels $\left.(\mathrm{a} / \mathrm{b})\right)$ is neutral $\left(\sigma_{r}=0.0\right)$ and its period is approximately $\left(T=\frac{L}{U^{*}} \frac{2 \pi}{\sigma_{i}}\right) 4.6$ months. The pattern shows a maximum response located to the north of the mid-axis which propagates westward. The second mode (Fig. 6(c/d)) has a period of about 1.5 year, it is also stable and its pattern resembles the first mode from the QG model (Figure 3) in that the perturbations propagate southwestward with the principle axis nearly orthogonal to the jet direction. The third mode (Fig. 6(e/f)), is slightly damped and has a similar period $(T=3.7$ months) as the first mode. In fact, the structures of the first and third mode appear symmetrically related.

The modes at the Hopf bifurcation $H_{2}$ are very similar to those at $H_{1}$. The ordering of the different modes with respect to their growth rate is different, with the first two modes having intermonthly period. The patterns for the first two modes are slightly displaced northward with respect to the patterns in Fig. 6(a/b) and Fig. 6(e/f). The pattern of the interannual mode, which is now the third mode with a period of 2.5 [yr], follows the orientation of the jet and its principle axis is again orthogonal to the direction of the jet.

At first sight, the bifurcation diagram in Fig. 5(a) appears in agreement with Fig. 2 in Speich et al. (1995). The solutions correspond very well, and also the positions of the Hopf bifurcations are very similar. However, closer inspection reveals that the branches are differently connected. In Speich et al. (1995), each solution on the branch existing for all values of $E$ has its confluence point displaced northwards, whereas in our case the confluence point is displaced southwards (e.g. Fig. 5(b)). A slightly different representation of friction in both models leads to a different imperfect pitchfork. 
In the results of Speich et al. (1995) the first mode at the Hopf bifurcation on the 'jet down' branch has an interannual period, whereas that on the 'jet up' branch has an intermonthly period. Although in our results the group of three modes is very close with respect to growth rate, the interannual mode appears only as second or third mode, while one of the intermonthly modes is most unstable. This indicates that small differences in formulation and discretisation may lead to a slightly different reordering of internal modes of variability. The interannual mode is therefore not robust as being the most dominant mode, but when this is the case, it destabilizes easily at slightly smaller friction. Although it is likely that the interannual mode plays a significant role in the flow dynamics, only a nonlinear analysis is able to demonstrate this.

\section{c. Relation between QG and SW results}

A close relation between the solutions of the QG and SW models exists. In Figure 7, the two bifurcation diagrams for the QG (drawn) and SW (dotted) model are replotted using the same control parameter within both models and the same norm $\phi$ of the solution. Note that the values of the other parameters were chosen in such a way that they exactly correspond and that for the QG-model, $\phi=\max (\psi L U H) \mathrm{Sv}$ and

$E=\frac{\epsilon U}{U^{*} R e}$. As is explained in more detail in the appendix, the QG-model admits a reflection symmetry through the mid-axis of the basin (Cessi and Ierley, 1995) and hence a pitchfork bifurcation is found. This symmetry is broken in the SW-model (Jiang et al., 1995), in a manner which is most clearly understood in the low forcing limit $\left(\alpha_{S} \rightarrow 0\right)$ as explained in the appendix.

In the limit of large $E$, the models have the same transport since they both become approximately linear. Small deviations from the equilibrium thermocline and advection induce an imperfection, illustrated by the break-up of the pitchfork bifurcation in the $\mathrm{SW}$-model. In the SW-model, the branch of the stable anti-symmetric double gyre solution (present in the QG-model) is connected continuously to one of the asymmetric solutions. The other asymmetric solution has connected to the branch originating from 
unstable part of the anti-symmetric solution in the QG-model, resulting in a limit point. It is clear that the occurrence of multiple equilibria in the SW-model has its origin in the symmetry breaking within the QG-model. The mechanism for this symmetry breaking was explained in detail in Dijkstra and Katsman (1997) and hence, applies in essence also to explain the multiple states in the SW-model. The exact way in which the pitchfork is perturbed (and hence which branch corresponds to the jet-up or jet-down solution) depends on a delicate balance between the different ageostrophic terms which can be analysed in the small forcing limit but is outside the scope of this paper.

Also a close relationship between the modes appearing as instabilities on the asymmetric solutions in both models exists. The patterns of the intermonthly modes in the QG-model (not shown) are slightly more symmetric and more localized in the jet than those in the SW-model. Their time scale is slightly shorter but they certainly deform into the intermonthly modes computed in the SW-model. The interannual mode in the QG-model hardly changes structure between both models. The ordering of the modes and their spectral separation (the distance between the growth rates of the modes) depends on the model formulation, is much larger in the QG-model and may have important impact on the time-dependent behavior of each model.

\section{Results: 'Realistic' case}

A realistically shaped basin $\Omega$ is defined by using data from a standard bathymetry data set. In Fig. 8, the finite element grid for the shallow water model is shown; this consists of 2489 triangular elements and amounts to a total of 11591 degrees of freedom. To keep a manageable set of equations for continuation, the basin size was slightly reduced (with respect to the real basin size) and the dimensions are about $2500 \times 2000$ kilometers, which may modify actual time scales of variability and volume transport with respect to reality. 
To represent a realistic wind forcing, the windstress was obtained from the Hellerman and Rosenstein (1983) data set. These data consist of values on a $2^{\circ} \times 2^{\circ}$ grid that is interpolated using a cubic spline fit to the finite element grid. The resulting windstress pattern is indicated by $\left(\tau_{H R}^{x}, \tau_{H R}^{y}\right)$.

\section{a. Multiple mean paths of the Gulf Stream}

For a maximum value of the windstress amplitude of $0.05 \mathrm{~Pa}$, which is slightly less than that in reality, the bifurcation diagram is shown in Figure 9 using $E$ as control parameter. Main result is the existence of multiple equilibria, just as in the rectangular geometry considered in the previous section. Two solution branches are found, on which solutions are already unstable for $E<7.510^{-6}$. One of the branches is connected to the large $E$ regime and a solution on this branch is shown for $E=3.010^{-6}$ in Figure $9(\mathrm{~b})$. The Gulf Stream passes along Cape Hatteras northward and near the North England Seamount Chain it turns into the open ocean. There is a very weak northern circulation region and at this value of $E$ the transport $\phi$ is about $21.5 \mathrm{~Sv}$, which is substantially smaller than current estimates near Cape Hatteras of about 50-65 Sv (Johns et al., $1995)$.

The other branch exists only for values of $E$ smaller than $3.210^{-6}$, which is the position of the limit point on this branch. The solution at $E=3.010^{-6}$ displays (Figure 9(c)) a Gulf Stream which turns into the open ocean near Cape Hatteras. There is now a strong northern circulation region, although too much concentrated near the coast compared to reality. At this value of $E$, the maximum transport of this 'separated' Gulf Stream is about 26.2 Sv, which is larger than that of the 'deflected' Gulf Stream (Figure 9(b)). The latitude and longitude at which the maximum in northward volume transport $\phi$ occurs are very similar and hence the separation behavior causes the different transport.

The first Hopf bifurcation is found at $E=7.510^{-6}$ on the branch of the deflected Gulf Stream solution ( $H$ in Figure $9(\mathrm{a})$ ). At $E=3.010^{-6}$, the deflected solution in 
Figure $9(\mathrm{~b})$ is unstable to only one oscillatory mode. The real and imaginary part of the eigenvector of this mode are shown in Figure $10(\mathrm{a} / \mathrm{b})$; the period of oscillation is about 5 months. It is clear that the center of action for the oscillation is located in the Gulf Stream. The scale of the perturbations is about $500 \mathrm{~km}$ and the principle axis makes about a $45^{\circ}$ angle with the $x$-axis. This orientation does not appear directly related to the orientation of the jet itself. The disturbances propagate south-westward against the flow direction of the mean current of the steady state. During propagation, the principle axis of the perturbations does not change much.

The separated solution (point (c) in Figure 9(a)) is also unstable to only one oscillatory mode (Figure 10(c/d)) having a period of about 6 months. Thickness perturbations with a scale of about $400 \mathrm{~km}$ are again localized in the jet. The centers of action are located at opposite centers of the jet and the response outside of the jet (e.g. for $x>-1$ ) is weak. Contrary to the unstable mode for the deflected solution, the orientation of the principle axis changes during one cycle of the oscillation which indicates that the perturbation moves around the jet in a clockwise manner.

Although both the bifurcation diagram as well as the structure of the modes of variability have similar properties than those in the rectangular geometry, this relation is explored in more detail below, by first 'deforming' the wind stress back to the simple form as in (11). Subsequently, one intermediate type of geometry between rectangular and 'realistic' is considered.

\section{b. Effect of windstress shape}

To study the transition of solutions from the Hellermann and Rosenstein windstress to the simple wind stress (11), a homotopy parameter $p_{h}$ is introduced that allows a continuous transition between the two different shapes of the wind-stress, according to

$$
\tau^{x}=\left(1-p_{h}\right) \tau_{S}^{x}+p_{h} \tau_{H R}^{x}
$$




$$
\tau^{y}=p_{h} \tau_{H R}^{y}
$$

In Fig. 11, the curl of the windstress field is shown for different values of the homotopy parameter $p_{h}$. For the simple windstress forcing $\tau_{S}\left(p_{h}=0\right)$, the change in sign of the curl of the windstress is located just above Cape Hatteras. When $p_{h}$ is increased, this zero contour rotates counterclockwise up to an angle of about $30^{\circ}$ at $p_{h}=1$.

The bifurcation diagram using $p_{h}$ as control parameter at fixed $E=7.610^{-6}$ is shown in Figure 12(a). From $p_{h}=1.0$ down to $p_{h}=0.3$, the transport increases nearly linearly due to the change in the windstress curl. For $0.3<p_{h}<0.6$, multiple steady states exist because two limit points appear and since the value of $E$ is quite large, two of these states are linearly stable; the third solution is unstable. The origin of these multiple equilibria is related to the imperfect pitchfork bifurcation since a different reconnection occurs around $p_{h}=0.5$. Hence, the jump in transport of the different solutions is connected to the different transports of the jet-up and jet-down solutions in the rectangular domain.

The two stable solutions for the same wind forcing $\left(p_{h}=0.5\right)$ are shown in panels $(\mathrm{b})$ and (c) of Figure 12. For one solution, separation of the Gulf Stream occurs near Cape Hatteras, whereas for the other solution, separation occurs at higher latitudes. The separated solution in Fig. 12(c) has a maximum northward transport of $41.5 \mathrm{~Sv}$ which is much larger than that of the deflected solution in Fig. 12(b) ( $\phi=32.2 \mathrm{~Sv})$. Again, on the upper branch, transport increases nearly linearly and for $p_{h}=0$, a Gulf Stream is found which separates nicely at Cape Hatteras and meanders into the North-Atlantic, with a maximum northward transport of about $80 \mathrm{~Sv}$.

The chosen value of $E$ in Fig. 12 corresponds to that of the first Hopf bifurcation for $p_{h}=0$ and hence, the solution in Fig. 12(d) is just critical. The corresponding eigenfunctions of the two most unstable modes are shown in Fig. 13. The period of the critical mode $\left(\sigma_{r}=0\right)$ is approximately 6 months (panels $\left.(\mathrm{a} / \mathrm{b})\right)$ and its pattern is similar to that of the first mode in the rectangular geometry (Fig. 6(a/b)). Its principle 
axis points in zonal direction and does not change much during the oscillation. The second oscillatory mode has a period of approximately 17 months (panels (c/d)). Both its interannual time scale and orientation of the principle axis correspond to that of the mode in Fig. 6(e/f) for the rectangular basin.

\section{c. Effect of geometry}

As a final step to demonstrate the connection between qualitative features within the hierarchy of models, the realistic domain is 'deformed' into a trapezium domain. The finite element grid for this domain is shown in Figure 14(a). The bifurcation diagram is similar to that in Fig. 9 and not shown. The steady solution for the simple wind forcing (11) at $E=10.110^{-6}$ (which is at the Hopf bifurcation for this case) is shown in Fig. 14(b). This solution resembles one of the asymmetric solutions of the rectangular basin, i.e. the 'jet down' solution, which is slightly deformed due to the geometry. Note that the value of $E$ at the critical boundary has increased, indicating that the solutions are more unstable than those in the rectangular basin. This is due to the larger basin size which induces a stronger jet. The solution is also very similar to Figure 12(d) obtained for realistic geometry and the windstress (11). Because the value of $E$ at the first Hopf bifurcation is smaller than that for the trapezium geometry, the effect of the realistic continental geometry is stabilizing.

The first two most unstable modes are shown at the Hopf bifurcation in Fig. 15 and again display the same type of features as those in the rectangular domain (Fig. 6). The first mode has an intermonthly period and resembles the mode in Fig. 6(a/b), whereas the second has an interannual period and resembles Fig. 6(c/d). Therefore, the order of intermonthly and interannual mode remains the same and this sequence of modes can be traced from simple to realistic geometry and windstress in the SW-model. 


\section{Summary and Discussion}

A close qualitative connection between steady solutions and modes of variability exists within a hierarchy of equivalent barotropic models. The model range is from a quasigeostrophic model in a rectangular basin forced by a cosine shaped zonal windstress to a shallow water model within a realistically shaped North-Atlantic basin forced by the Hellerman and Rosenstein windstress. The main result is the existence of multiple equilibria in the latter model, with two different mean states for the Gulf Stream path.

The dynamical origin of these multiple mean paths can be understood by looking at the changes in the bifurcation diagram through the model hierarchy. The unforced quasi-geostrophic equations in a rectangular geometry are invariant with respect to a reflection symmetry, because the balances of the geostrophic and ageostrophic terms, each group having a different symmetry, are considered separately. If the windstress has a reflection symmetry, such as the idealized windstress (11), then symmetry breaking of the resulting flow occurs through a pitchfork bifurcation. The physical mechanism of the symmetry breaking is easily understood from the perturbation structure at bifurcation (Dijkstra and Katsman, 1997). It is barotropic in nature and involves an asymmetric weakening/strengthening of the subpolar/subtropical gyre with a positive feedback from the resulting change in horizontal shear. The symmetry breaking leads to multiple equilibria and asymmetric solutions, and the latter solutions are related through the reflection symmetry. These asymmetric solutions are destabilized at Hopf bifurcations through basin modes, having an intermonthly period, and gyre modes, having an interannual period (Dijkstra and Katsman, 1997).

In the shallow water formulation for the rectangular basin, the balances of geostrophic and ageostrophic terms are not separated and consequently, the equations are not invariant with respect to the reflection symmetry. This was demonstrated in the low forcing limit $\alpha_{S} \rightarrow 0$, where for the velocity field the geostrophic and ageostrophic 
balances are decoupled up to $\mathcal{O}\left(\alpha_{S}\right)$ (see the appendix). However, the solution of the thermocline depth is not invariant under the reflection and hence even with the idealized windstress (11) no perfect pitchfork bifurcation is found. Instead, an imperfection of this structure is found, with the consequence that the multiple equilibria still exist, but that the solutions on each branch are no longer exactly symmetrically related.

In deforming the rectangular basin to the realistic geometry of the North-Atlantic basin, and the forcing into a realistic windstress pattern, the imperfect pitchfork structure remains robust. The 'jet up' and 'jet down' solution, i.e. the multiple equilibria in the simple case, deform into solutions with different separation behavior near the North-American coast of the Gulf Stream for the realistic case. No new equilibria are introduced by the change in geometry, which strongly suggest that the simultaneous existence of the deflected and separated Gulf Stream is not due to continental geometry. This is supported by results of intermediate cases, such as a trapezium geometry and a realistically shaped basin forced by the windstress (11).

The existence of the two solutions can be traced back as being caused by internal ocean dynamics, i.e. the existence of symmetry breaking in the highly idealized case. This result is at first sight surprising, since one would think that geometry would have a strong impact on the flow. Of course there is a quantitative dependence, since the patterns of both steady states and the modes of variability change and adapt to the geometry. Qualitatively, however, the dynamics is strongly controlled by the internal dynamics and no new changes are introduced by the geometry. In a way, the symmetry breaking observed in the QG model in rectangular geometry is still present, but it is localized in the real continental geometry and the extent of imperfection is not controlled by the overall details of the geometry, but by the local dynamics (i.e. jet structure) of the flow. Of course, the jet structure itself is controlled by boundary conditions and the values of the parameters in the model.

This also explains why the modes of variability are closely linked within the hier- 
archy of the models. In the range of parameters studied, there are only a few modes which can contribute to the dynamics; these modes easily extract energy from the basic state. Among those are intermonthly modes and interannual modes and their actual importance depends on the spectral separation, which is larger in the QG-model than in the SW-model. Although the actual ordering of the modes is sensitive to details in the model, it are only these type of modes which appear through the whole hierarchy. The time scale of these modes does not depend much on the continental geometry and windstress and even their pattern is not strongly modified. The reason is that already in rectangular geometry, these modes are strongly localized within the jet, which basically does not change through the range of different models.

Since multiple equilibria exist in these models, it is not unlikely that these play a role in the problem of Gulf Stream separation in realistic models. Preliminary computed trajectories, within the most realistic model used here, show that if one starts on the separated solution near Figure 9(b), the trajectory is first attracted towards a periodic orbit associated with the oscillatory unstable mode on this state. However, after some time it moves away from this orbit and finally arrives at a stable periodic orbit around the deflected solution. The periodic orbit coming out of the Hopf bifurcation on the branch which is continuously connected to the large $E$ solution (Figure $9(\mathrm{a})$ ), appears more stable than that on the isolated branch. The connection within the imperfect pitchfork is central to get on the right solution branch. For the shallow water in the rectangular basin case this connection is very sensitive to details in parameterization of subgrid scale processes. It is already different between the model used here and that in Speich et al. (1995), which only differs slightly in the parameterization of lateral friction.

Even if the steady solution branches are unstable to a few other type of instabilities (i.e. baroclinic instabilities) they may still influence the mean state, since they are still stable to many other modes. A delicate balance in the western boundary region may 
therefore determine whether the right separated Gulf Stream will be obtained as mean state in a simulation of the circulation. Since this delicate balance is easily influenced by all kinds of (even minor) phenomena, this may be the reason that a multitude of mechanisms has been proposed to be important for separation. Of course, further work on this delicate balance is needed and the low forcing limit is an obvious limit to consider this problem in more detail, since it is here that the connection is already determined.

The use of continuation methods has been essential in obtaining the correspondence between qualitative behavior through a hierarchy of models. For transient codes, this correspondence would always remain hidden because many of the steady states are unstable, mostly to time periodic perturbations. Hence, they would not be seen by the results of these models, although features of the steady states would appear in the trajectories. The results presented here give support for the relevance of studies using models in idealized geometry. Although certainly these models have their limitations with respect to quantitative prediction of phenomena in the real system, the results have more than only conceptual value, since the correct qualitative dynamics of the flow is captured. This is important knowledge when tackling hard problems such as the separation problem of western boundary currents with simple models and may temper critique on these studies related to the simplifications made. In fact, the strong qualitative correspondence found here may be a reason why studies with simple models have indeed contributed to improve aspects of OGCM's.

\section{Acknowledgements}

This work was supported by the Netherlands Organization for Scientific Research (NWO) under a PIONIER grant to HD. All computations were performed on the CRAY C916 at the Academic Computing Centre (SARA), Amsterdam, the Netherlands within the project SC498. Use of these computing facilities was sponsored by the National Com- 
puting Facilities Foundation with financial support from NWO. 


\section{Appendix: Symmetry properties of QG- and SW-equations}

The unforced quasi-geostrophic model admits a reflection symmetry through the mid-axis of the basin (Cessi and Ierley, 1995). This reflection $\mathcal{R}_{Q G}$ has the representation

$$
\mathcal{R}_{Q S}(\psi(x, A-y), \zeta(x, A-y))=-(\psi(x, y), \zeta(x, y))
$$

If the applied windstress forcing is symmetric, there are constraints on the type of bifurcation points. For the idealized windstress forcing such as in (11), a symmetry breaking pitchfork is the generic bifurcation (Golubitsky and Schaeffer, 1985).

The appropriate reflection symmetry in the shallow water model is represented by

$$
\mathcal{R}_{S W}(u(x, A-y), v(x, A-y), h(x, A-y))=(u(x, y),-v(x, y),-h(x, y))
$$

It can easily be shown that the SW equations (even with the idealized windstress (11)) are not invariant with respect to the reflection $\mathcal{R}_{S W}$. Symmetry is not only broken by advection, but also by other ageostrophic effects, such as the thermocline deviations from their equilibrium value. This asymmetry is therefore already present in the low forcing limit, i.e. $\alpha_{S} \rightarrow 0$. If we expand quantities as

$$
\begin{aligned}
& u=\alpha_{S} u^{(1)}+\alpha_{S}{ }^{2} u^{(2)}+\mathcal{O}\left(\alpha_{S}{ }^{3}\right) \\
& v=\alpha_{S} v^{(1)}+\alpha_{S}^{2} v^{(2)}+\mathcal{O}\left(\alpha_{S}^{3}\right) \\
& h=1+\alpha_{S} h^{(1)}+\alpha_{S}{ }^{2} h^{(2)}+\mathcal{O}\left(\alpha_{S}^{3}\right)
\end{aligned}
$$

the steady equations at $\mathcal{O}\left(\alpha_{S}\right)$ (with $\tau^{y}=0, r=0$ and $\tau^{x}=\tau^{x}(y)$ ) are

$$
-(1+\epsilon \beta y) v^{(1)}=-\epsilon F^{*} \frac{\partial h^{(1)}}{\partial x}+E \nabla^{2} u^{(1)}+\tau^{x}
$$




$$
\begin{aligned}
(1+\epsilon \beta y) u^{(1)} & =-\epsilon F^{*} \frac{\partial h^{(1)}}{\partial y}+E \nabla^{2} v^{(1)} \\
\frac{\partial u^{(1)}}{\partial x}+\frac{\partial v^{(1)}}{\partial y} & =0
\end{aligned}
$$

When $h^{(1)}$ is eliminated, we obtain the (Munk) problem

$$
\beta v^{(1)}=\frac{1}{R e} \nabla^{2} \zeta^{(1)}-\frac{1}{\epsilon} \frac{\partial \tau^{x}}{\partial y}
$$

where $\zeta^{(1)}=\frac{\partial v^{(1)}}{\partial x}-\frac{\partial u^{(1)}}{\partial y}$.

When $\tau^{x}$ is even with respect to $A / 2$, the solutions for $u^{(1)}$ and $v^{(1)}$ are even and odd, respectively, and hence invariant to (16). However, when the divergence of the first two equations (18) are taken, the equation for the thermocline anomaly becomes

$$
\epsilon F^{*} \nabla^{2} h^{(1)}=(1+\epsilon \beta y) \zeta^{(1)}-\epsilon \beta u^{(1)}
$$

which can (in principle) be solved using appropriate boundary conditions. Since $\zeta^{(1)}$ is odd and $u^{(1)}$ is even, $h^{(1)}$ cannot be odd and the symmetry (16) is broken. Hence, no perfect pitchfork bifurcation can occur and an imperfect one results. 


\section{Captions to the Figures}

\section{Figure 1}

Bifurcation diagram for the 1.5 layer QG model using the Reynolds number as control parameter. On the vertical axis, the quantity $\Psi=\max \psi+\min \psi$ is plotted. Drawn (dotted) branches indicate (unstable) stable states, whereas bifurcation points are indicated by markers.

\section{Figure 2}

Asymmetric solution of the QG-model at Re $=67$, just above the Hopf bifurcation $H$ on the lower branch in Figure 1.

\section{Figure 3}

Unstable eigenmode at $R e=67$, which is slightly above the Hopf bifurcation $H$. The eigenvalue at this point is $\sigma_{r}=5.9810^{-2}$ and $\sigma_{i}=2.87$ the latter corresponding to a period $T=6.9[y r]$. The mode represented by contour plots of the streamfunction $\psi$ of (a) Real part of the eigenvector. (b) Imaginary part of the eigenvector.

\section{Figure 4}

Finite element grid used for computations with the shallow water model on a rectangular domain.

\section{Figure 5}

(a) Bifurcation diagram using the Ekman number $E$ as control parameter for the shallow water model in the rectangular domain. On the vertical axis, the volume transport $\phi$ as in (13) is plotted. (b) Pattern of the layer thickness anomaly of the steady-state solution at the Hopf bifurction $H_{1}\left(E=0.3410^{-5}\right)$. (c) As in (b) but at the Hopf bifurcation $H_{2}\left(E=0.3610^{-5}\right)$. 


\section{Figure 6}

First three eigenmodes at the Hopf bifurcation $H_{1}\left(E=0.3410^{-5}\right)$. (a/b) Layer thickness perturbations for the real and imaginary part of the most unstable mode with $\sigma_{r}=$ 0.0 and $\sigma_{i}=0.52$. (c/d) As $(\mathrm{a} / \mathrm{b})$ but for the 2 nd mode $\left(\sigma_{r}=-3.610^{-2}, \sigma_{i}=0.13\right)$. (e/f) As (a/b) but for the 3rd mode $\left(\sigma_{r}=-4.210^{-2}, \sigma_{i}=0.65\right)$.

\section{Figure 7}

Bifurcation diagram for both the QG-model (drawn) and SW-model (dotted) in a rectangular basin using the Ekman number $E$ as control parameter.

\section{Figure 8}

Finite element grid used for computations on a realistically shaped North-Atlantic basin from $10^{\circ} \mathrm{N}$ to $60^{\circ} \mathrm{N}$.

\section{Figure 9}

Bifurcation diagram for the realistic case, using the Ekman number as control parameter; drawn (dotted) branches indicate stable (unstable) steady states. (b) Contour plot of the layer thickness anomaly for the 'deflected' Gulf Stream at $E=3.0 \times 10^{-6}$. (c) Contour plot of the layer thickness anomaly for the 'separated' Gulf Stream at $E=3.0 \times 10^{-6}$.

\section{Figure 10}

Eigenfunctions corresponding to the unstable mode at the marked points in Figure 9(a) for $E=3.010^{-6}$; shown are the layer thickness perturbations. (a/b): Real and imaginary part of the unstable oscillatory mode on the steady state shown in Figure 9(b), with $\sigma_{r}=0.71 \times 10^{-1}$ and $\sigma_{i}=0.51$. (c/d): As in $(\mathrm{a} / \mathrm{b})$ but for steady state shown in 
Figure $9(\mathrm{c})$, with $\sigma_{r}=0.15 \times 10^{-1}$ and $\sigma_{i}=0.61$.

\section{Figure 11}

Curl of windstress (14) for three different values of $p_{h}$. All panels are scaled with respect to the same maximum amplitude. a) $p_{h}=0.0$. b) $p_{h}=0.5$. c) $p_{h}=1.0$.

\section{Figure 12}

Bifurcation diagram using the homotopy parameter $p_{h}$ as control parameter to continue from simple wind forcing (11) to the Hellermann and Rosenstein forcing $p_{h}=1.0$ at $E=7.610^{-6}$. (b) Layer thickness anomalies for the steady state at $p_{h}=0.5(\phi=$ $41.5 \mathrm{~Sv})$. (c) Layer thickness anomalies for the steady state at $p_{h}=0.5(\phi=32.2 \mathrm{~Sv})$. (d) Layer thickness anomalies for the steady state at $p_{h}=0.0(\phi=80.0 \mathrm{~Sv})$.

\section{Figure 13}

Eigenfunctions at the first Hopf bifurcation $\left(E=7.610^{-6}\right)$ for the windstress forcing (11), i.e. $p_{h}=0$. $(\mathrm{a} / \mathrm{b})$ : Layer thickness perturbations for the real and imaginary part of first eigenmode, with $\sigma_{r}=0.0$ and $\sigma_{i}=0.43$. (c/d): As in (a/b) but for the second mode, with $\sigma_{r}=-0.1010^{-3}$ and $\sigma_{i}=0.14$.

\section{Figure 14}

a) Finite element grid used for computations on the trapezium domain. b) Basic state at the first Hopf bifurcation at $E=10.110^{-6}$.

\section{Figure 15}

Eigenfunction at the first Hopf bifurcation $\left(E=10.110^{-6}\right)$ for the trapezium domain and $p_{h}=0$. (a/b): Layer thickness perturbations of the real en imaginary part of the first eigenmode with $\sigma_{r}=0.0$ and $\sigma_{i}=0.42$. (c/d): Layer thickness perturbations of 
the real en imaginary part of the second eigenmode with $\sigma_{r}=-0.018$ and $\sigma_{i}=0.18$. 


\section{References}

Beckmann, A., Böning, C. W., Köberle, J., and Willebrand, J. (1994). Effects of increased horizontal resolution in a simulation of the North-Atlantic ocean. J. Phys. Oceanogr., 24, 326-344.

Berloff, P. and Meacham, S. (1997). The dynamics of an equivalent barotropic model of the wind-driven circulation. J. Mar. Res., 55, 407-451.

Bryan, F. O., Böning, C. W., and Holland, W. (1995). On the midlatitude circulation in a high-resolution model of the North-Atlantic. J. Phys. Oceanogr., 25, 289-305.

Cessi, P. and Ierley, G. R. (1995). Symmetry-breaking multiple equilibria in quasigeostrophic, wind-driven flows. J. Phys. Oceanogr., 25, 1196-1205.

Cessi, P., Vance Condie, R., and Young, W. R. (1995). Dissipative dynamics of western boundary currents. J. Phys. Oceanogr., 25, 1196-1205.

Chassignet, E. P. and Bleck, R. (1993). The influence of layer outcropping on the separation of boundary currents. Part I: The wind-driven experiments. J. Phys. Oceanogr., 23, 1485-1507.

Dengg, J. (1993). The problem of Gulf Stream separation: A barotropic approach. J. Phys. Oceanogr., 23, 2182-2200.

Dengg, J., A., B., and Gerdes, R. (1996). The Gulf Stream separation problem. In W. Kraus, editor, The warmwatersphere of the North Atlantic Ocean, pages 253-290. Borntraeger.

Dijkstra, H. A. and Katsman, C. A. (1997). Temporal variability of the wind-driven quasi-geostrophic double gyre ocean circulation: Basic bifurcation diagrams. Geophys. Astrophys. Fluid Dyn., 85, 195-232. 
Dijkstra, H. A., Molemaker, M. J., Van der Ploeg, A. J., and Botta, E. F. F. (1995). An efficient code to compute nonparallel flows and their linear stability. Comp. Fluids, 24, 415-434.

Fofonoff, N. P. (1954). Steady flow in a frictionless homogeneous ocean. J. Mar. Res., 13, 254-262.

Gangopadhyay, A., Cornillon, P., and Watts, D. R. (1992). A test of the ParsonsVeronis hypothesis on the separation of the Gulf Stream. J. Phys. Oceanogr., 22, $1286-1301$.

Golubitsky, M. and Schaeffer, D. G. (1985). Singularities and groups in bifurcation theory. Springer-Verlag.

Guckenheimer, J. and Holmes, P. (1983). Nonlinear oscillations, dynamical systems and bifurcations of vector fields. Springer-Verlag.

Hellerman, S. and Rosenstein, M. (1983). Normal monthly wind stress over the world ocean with error estimates. J. Phys. Oceanogr., 13, 1093-1104.

Holland, W. and Lin, L. (1975). On the generation of mesoscale eddies and their contribution to the ocean general circulation. J. Phys. Oceanogr., 5, 642-669.

Holland, W. R. (1978). The role of mesoscale eddies in the general circulation of the ocean - numerical experiments using a quasi-geostrophic model. J. Phys. Oceanogr., 8, 363-392.

Holland, W. R. and Bryan, F. (1994). Modelling the wind and thermohaline circulation in the North-Atlantic Ocean. In P. Malanotte-Rizzoli and A. Robinson, editors, Ocean processes in Climate Dynamics: Global and Mediterranean examples, pages 35-156. Kluwer Academic, Netherlands. 
Holland, W. R. and Schmitz, W. J. (1985). Zonal penetration scale of model midlatitude jets. J. Phys. Oceanogr., 15, 1859-1875.

Jiang, S., Jin, F. F., and Ghil, M. (1995). Multiple equilibria and aperiodic solutions in a wind-driven double gyre, shallow water model. J. Phys. Oceanogr., 25, 764-786.

Johns, W. E., Shay, T. J., Bane, J. M., and Watts, D. R. (1995). Gulf Stream structure, transport and recirculation near $68^{\circ}$ w. J. Geoph. Res., 100, 817-838.

McWilliams, J. C. (1996). Modeling the ocean general circulation. Ann. Rev. Fluid Mechanics, 28, 215-248.

Meacham, S. and Berloff, P. (1998). Barotropic, wind-driven circulation in a small basin. J. Mar. Res., 55, 523-563.

Meyers, P. G., Fanning, A. F., and Weaver, A. (1996). JEBAR, bottom pressure torque and Gulf Stream separation. J. Phys. Oceanogr., 26, 671-683.

Miller, A., Holland, W., and Hendershott, M. (1987). Open - ocean response and normal mode exitation in an eddy-resolving general circulation model. Geophys. Astrophys. Fluid Dyn., 37, 253-278.

Molemaker, M. and Dijkstra, H. (1998). Multiple equilibria and stability of the NorthAtlantic wind-driven ocean circulation. IMA Journal of Numerical Analysis, in press.

Munk, W. (1950). On the wind-driven ocean circulation. J. Meteor., 7, 79-93.

New, A. L., Bleck, R., Jia, Y., Marsh, M., Huddleston, M., and Barnard, S. (1996). An isopycnic model study of the North Atlantic. Part 1: Model experiment and water mass formation. J. Phys. Oceanogr., 26, 253-278.

Pedlosky, J. (1987). Geophysical Fluid Dynamics. Academinc Press, New York, U.S.A. 
Pedlosky, J. (1996). Ocean Circulation Theory. Springer, New York, Berlin.

Schmitz, W. J. and Holland, W. R. (1982). A preliminary comparison of selected numerical eddy-resolving general circulation experiments with observations. J. Mar. Res., 40, 75-117.

Semtner, A. J. and Chervin, R. M. (1992). Ocean general circulation from a global eddy-resolving model. J. Geoph. Res., 97, 5493-5550.

Speich, S., Dijkstra, H. A., and Ghil, M. (1995). Successive bifurcations in a shallow water model, applied to the wind-driven ocean circulation. Nonl. Proc. Geophys., 37, 289-306.

Stern, M. (1975). Minimal properties of planetary eddies. J. Mar. Res., 33, 1-13.

Stommel, H. (1948). The westward intensification of wind-driven ocean currents. Trans. Amer. Geophysical Union, 29, 202-206.

Van der Vaart, P. C. F. and Dijkstra, H. A. (1997). Sideband instabilities of mixed barotropic/baroclinic waves growing on a midlatitude zonal jet. Phys. Fluids, 9, 615-631. 
Dimensional parameters

$\begin{array}{cccc}\text { Parameter } & \text { Value } & \text { Parameter } & \text { Value } \\ L & 1.0 \times 10^{6} \mathrm{~m} & \tau_{0} & 5.0 \times 10^{-2} \mathrm{~Pa} \\ D & 5.0 \times 10^{2} \mathrm{~m} & \beta_{0} & 2.0 \times 10^{-11}(\mathrm{~ms})^{-1} \\ f_{0} & 5.0 \times 10^{-5} \mathrm{~s}^{-1} & A_{H} & 2.0 \times 10^{2} \mathrm{~m}^{2} \mathrm{~s}^{-1} \\ g^{\prime} & 3.0 \times 10^{-2} \mathrm{~ms}^{-2} & U & 1.0 \times 10^{-2} \mathrm{~ms}^{-1} \\ \rho_{0} & 1.0 \times 10^{3} \mathrm{kgm}^{-3} & \epsilon_{0} & 5.0 \times 10^{-8} \mathrm{~s}^{-1}\end{array}$

Dimensionless parameters

$\begin{array}{cccc}\text { Parameter } & \text { Value } & \text { Parameter } & \text { Value } \\ \beta & 2.0 \times 10^{3} & R e & 5.0 \times 10^{1} \\ \alpha_{Q} & 1.0 \times 10^{3} & F & 1.7 \times 10^{2} \\ r & 5.0 & & \end{array}$

Table 1: Standard values of parameters in the 1.5 layer $Q G$ model 
Dimensional parameters

$\begin{array}{cccc}\text { Parameter } & \text { Value } & \text { Parameter } & \text { Value } \\ L & 1.0 \times 10^{6} \mathrm{~m} & \tau_{0} & 5.0 \times 10^{-2} \mathrm{~Pa} \\ D & 5.0 \times 10^{2} \mathrm{~m} & \beta_{0} & 2.0 \times 10^{-11}(\mathrm{~ms})^{-1} \\ f_{0} & 5.0 \times 10^{-5} \mathrm{~s}^{-1} & A_{H} & 2.0 \times 10^{2} \mathrm{~m}^{2} \mathrm{~s}^{-1} \\ g^{\prime} & 3.0 \times 10^{-2} \mathrm{~ms}^{-2} & U^{*} & 1.0 \mathrm{~ms}^{-1} \\ \rho_{0} & 1.0 \times 10^{3} \mathrm{kgm}^{-3} & \epsilon_{0} & 5.0 \times 10^{-8} \mathrm{~s}^{-1}\end{array}$

Dimensionless parameters

$\begin{array}{cccc}\text { Parameter } & \text { Value } & \text { Parameter } & \text { Value } \\ \beta & 2.0 \times 10^{1} & E & 4.0 \times 10^{-6} \\ \epsilon & 2.0 \times 10^{-2} & F & 1.5 \times 10^{1} \\ r & 1.0 \times 10^{-3} & \alpha_{S} & 2.0 \times 10^{-3}\end{array}$

Table 2: Standard values of parameters in the 1.5 layer $S W$ model 


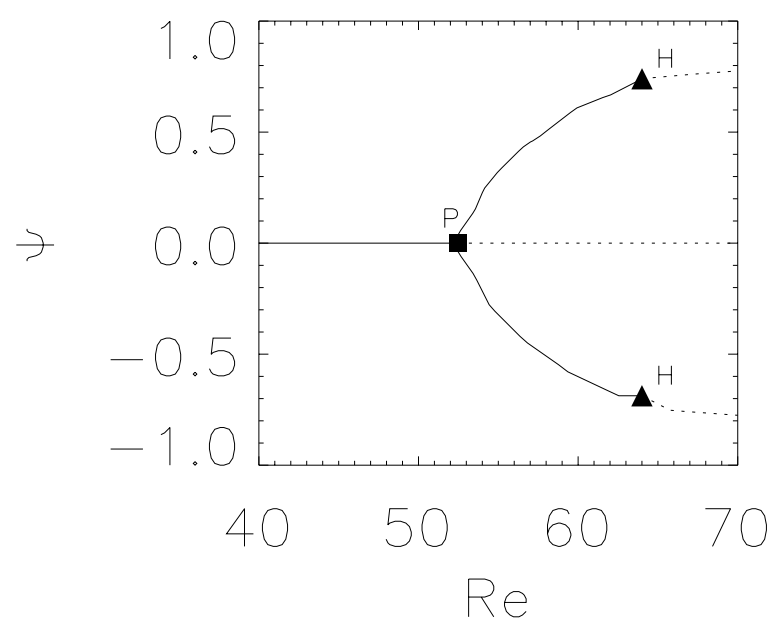

Figure 1: H.A. Dijkstra and M.J. Molemaker 


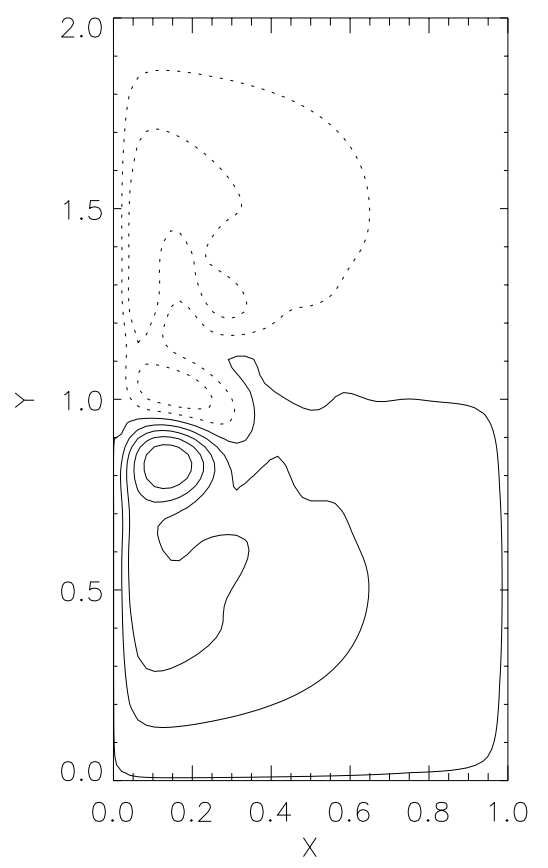

Figure 2: H.A. Dijkstra and M.J. Molemaker 


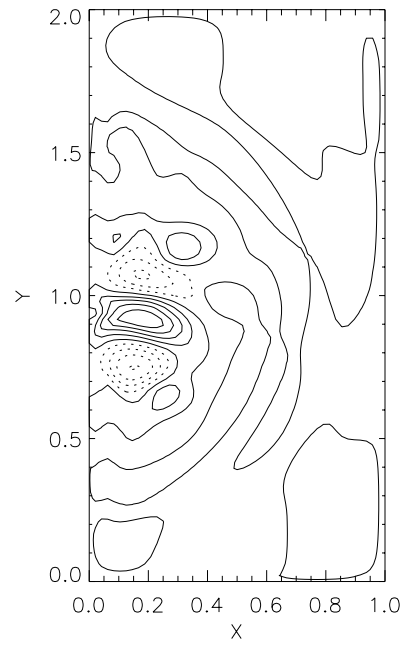

(a)

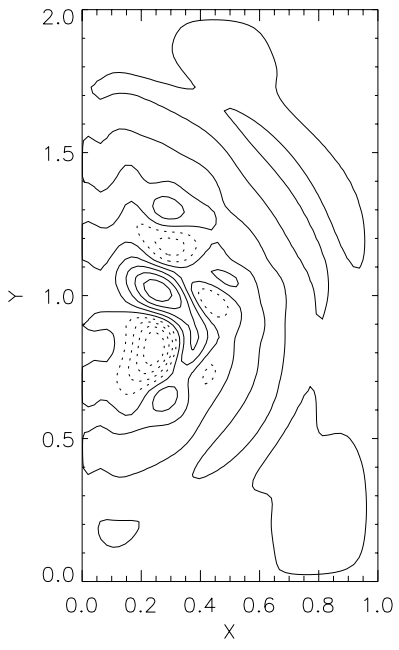

(b)

Figure 3: H.A. Dijkstra and M.J. Molemaker 


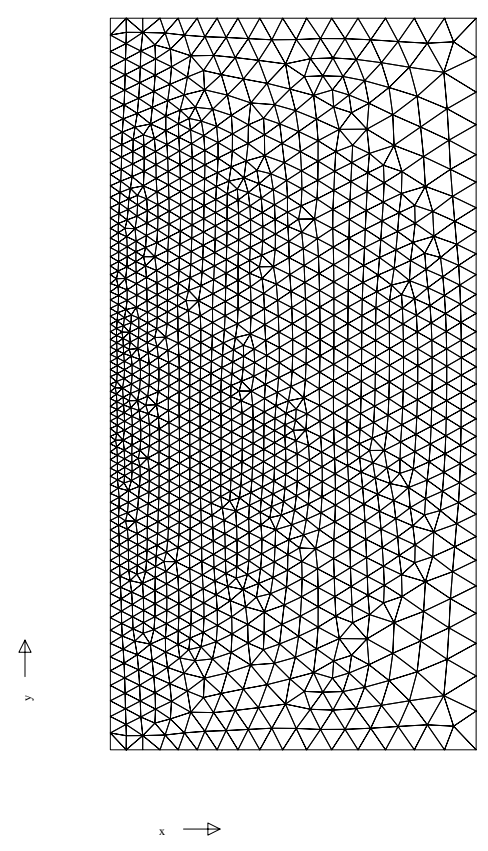

Figure 4: H.A. Dijkstra and M.J. Molemaker 


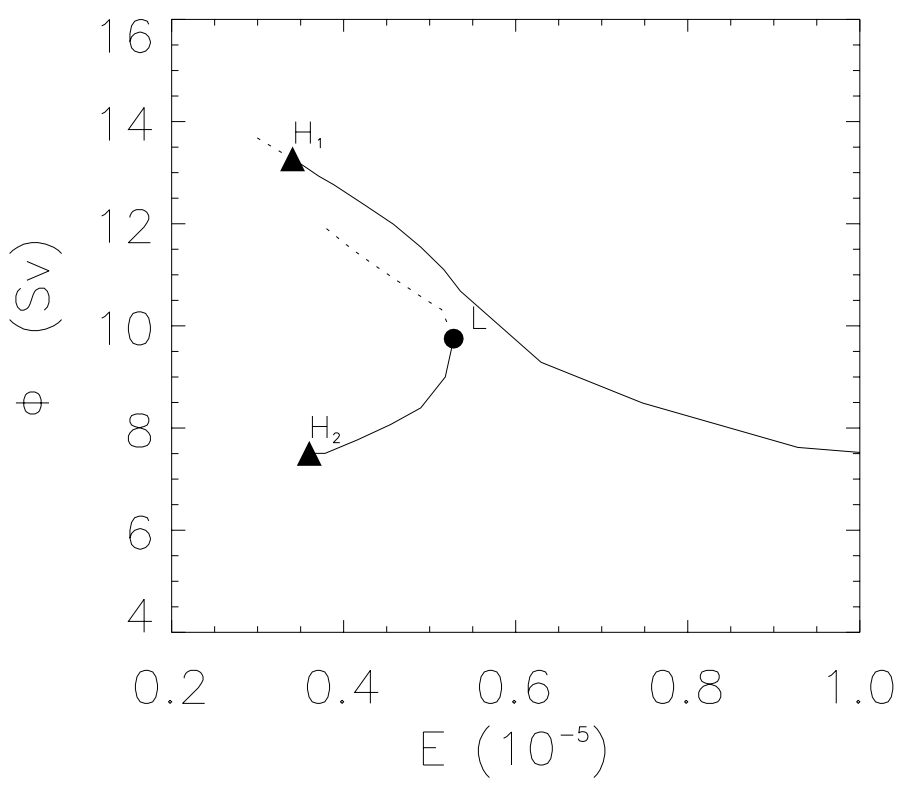

(a)

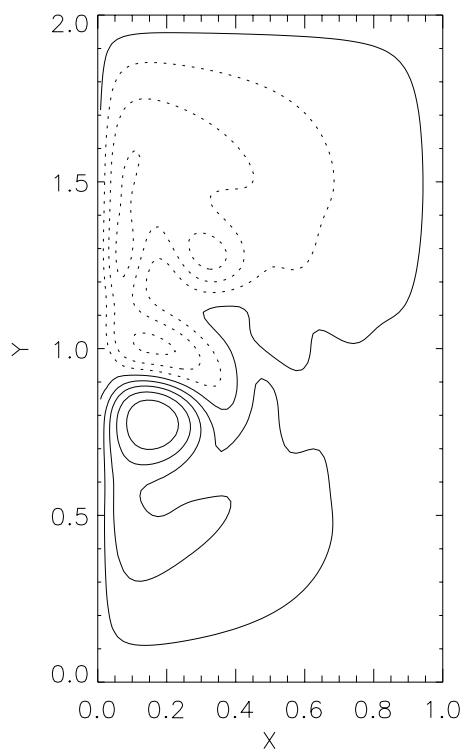

(b)

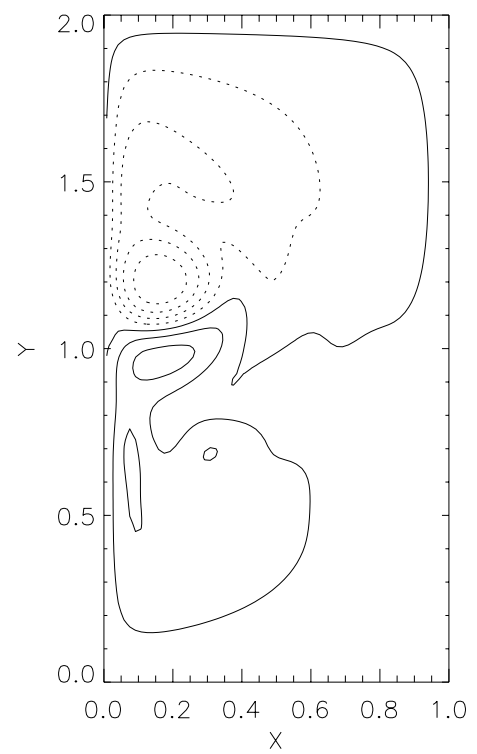

(c)

Figure 5: H.A. Dijkstra and M.J. Molemaker 
(a)
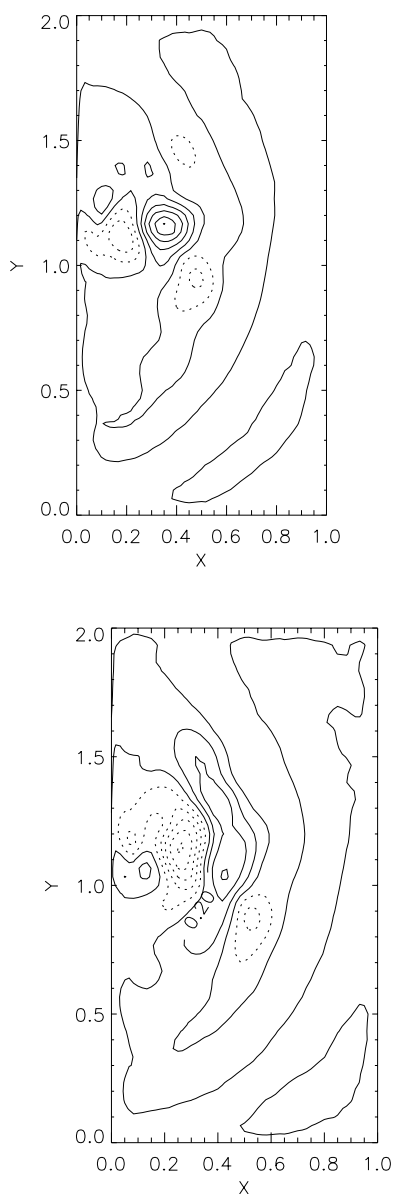

(b)

(c)
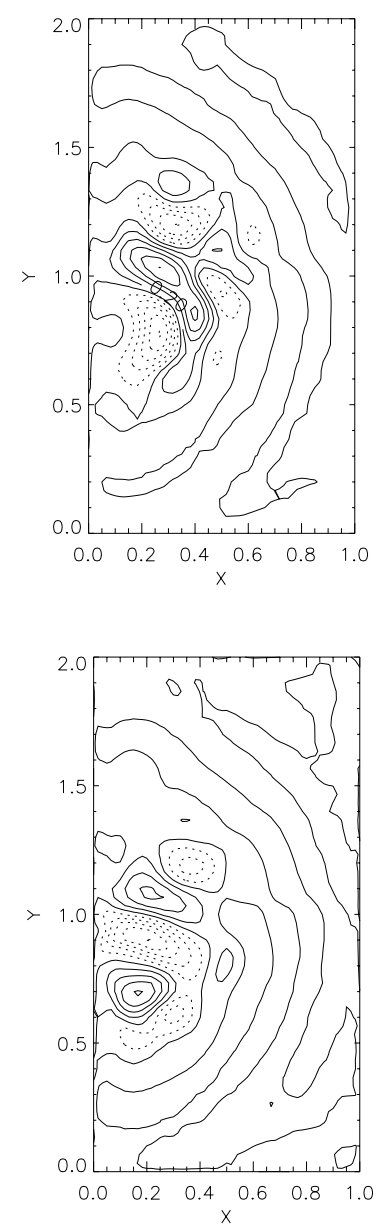

(d)

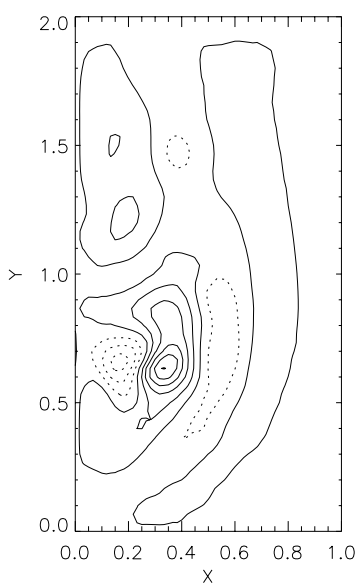

(e)

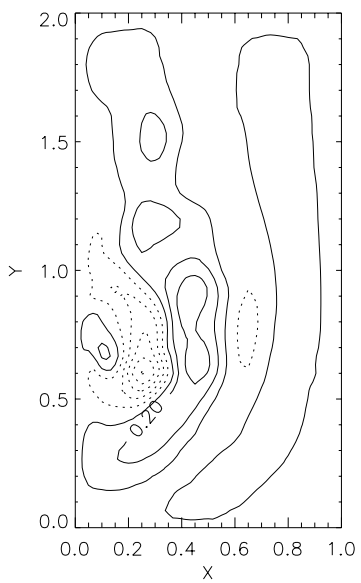

(f)

Figure 6: H.A. Dijkstra and M.J. Molemaker 


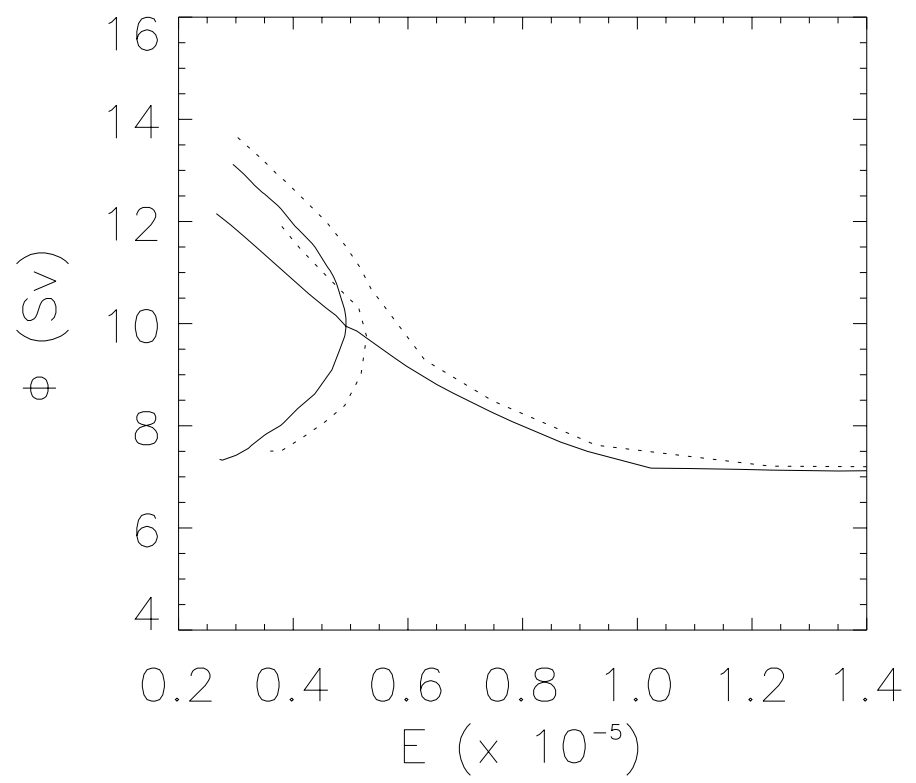

Figure 7: H.A. Dijkstra and M.J. Molemaker

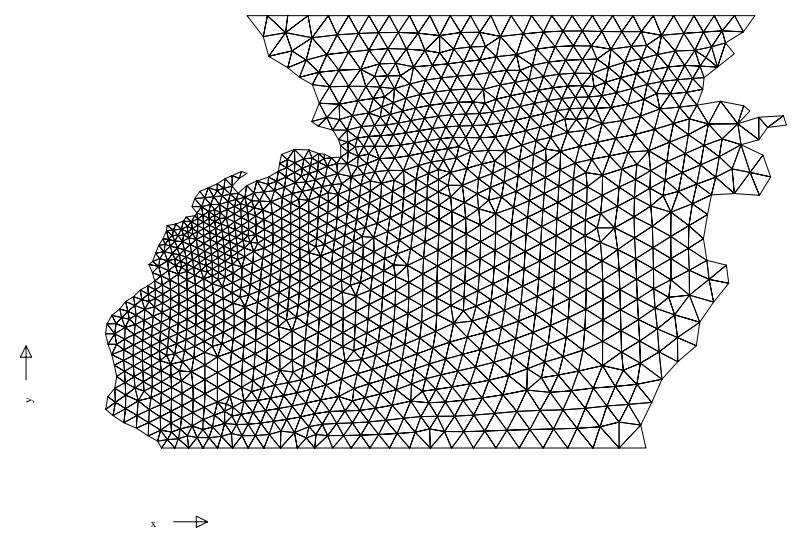

Figure 8: H.A. Dijkstra and M.J. Molemaker 


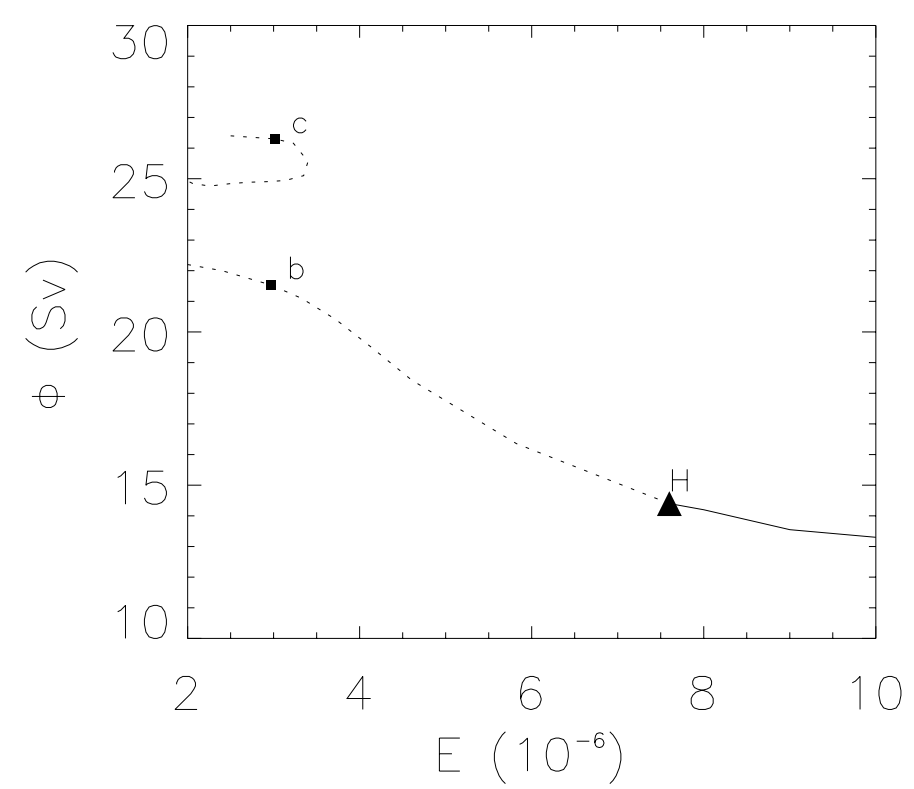

(a)

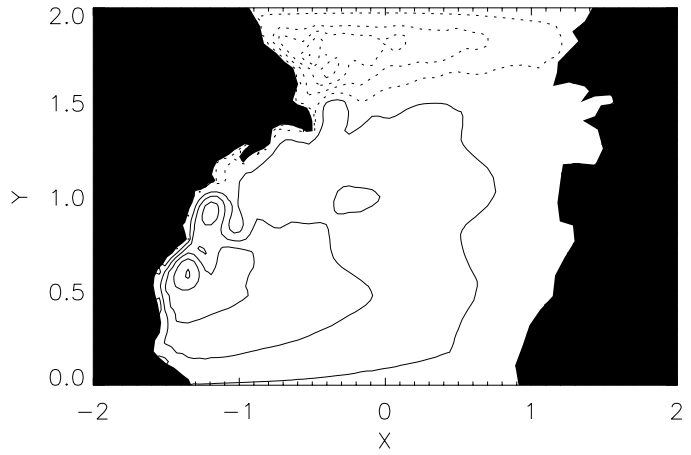

(b)

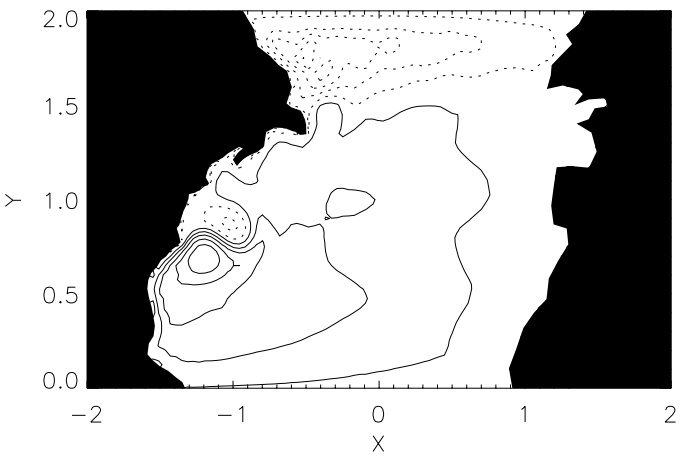

(c)

Figure 9: H.A. Dijkstra and M.J. Molemaker 


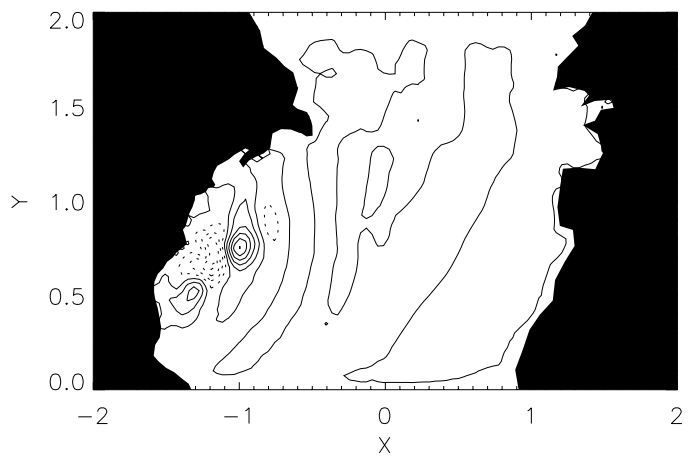

(a)

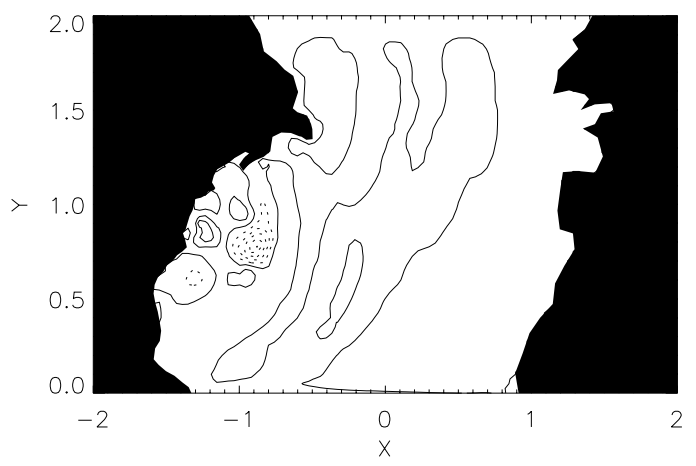

(c)

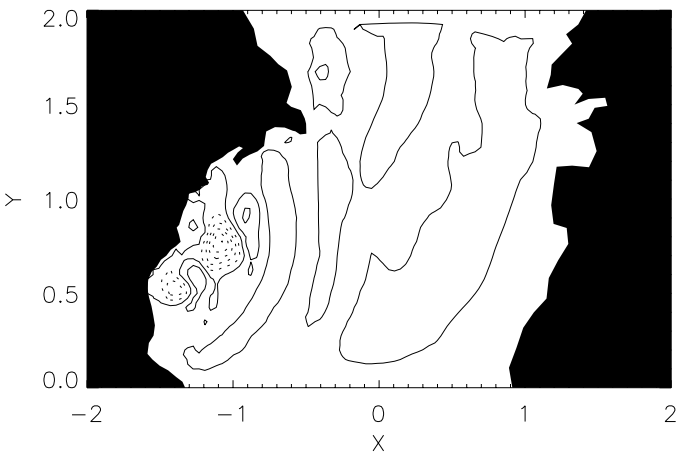

(b)

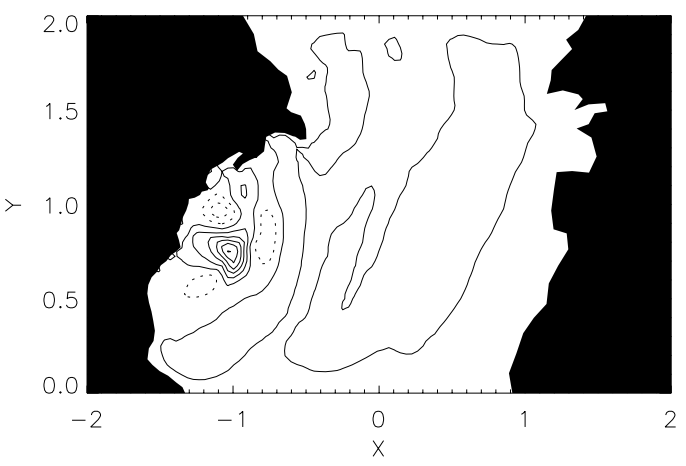

(d)

Figure 10: H.A. Dijkstra and M.J. Molemaker 

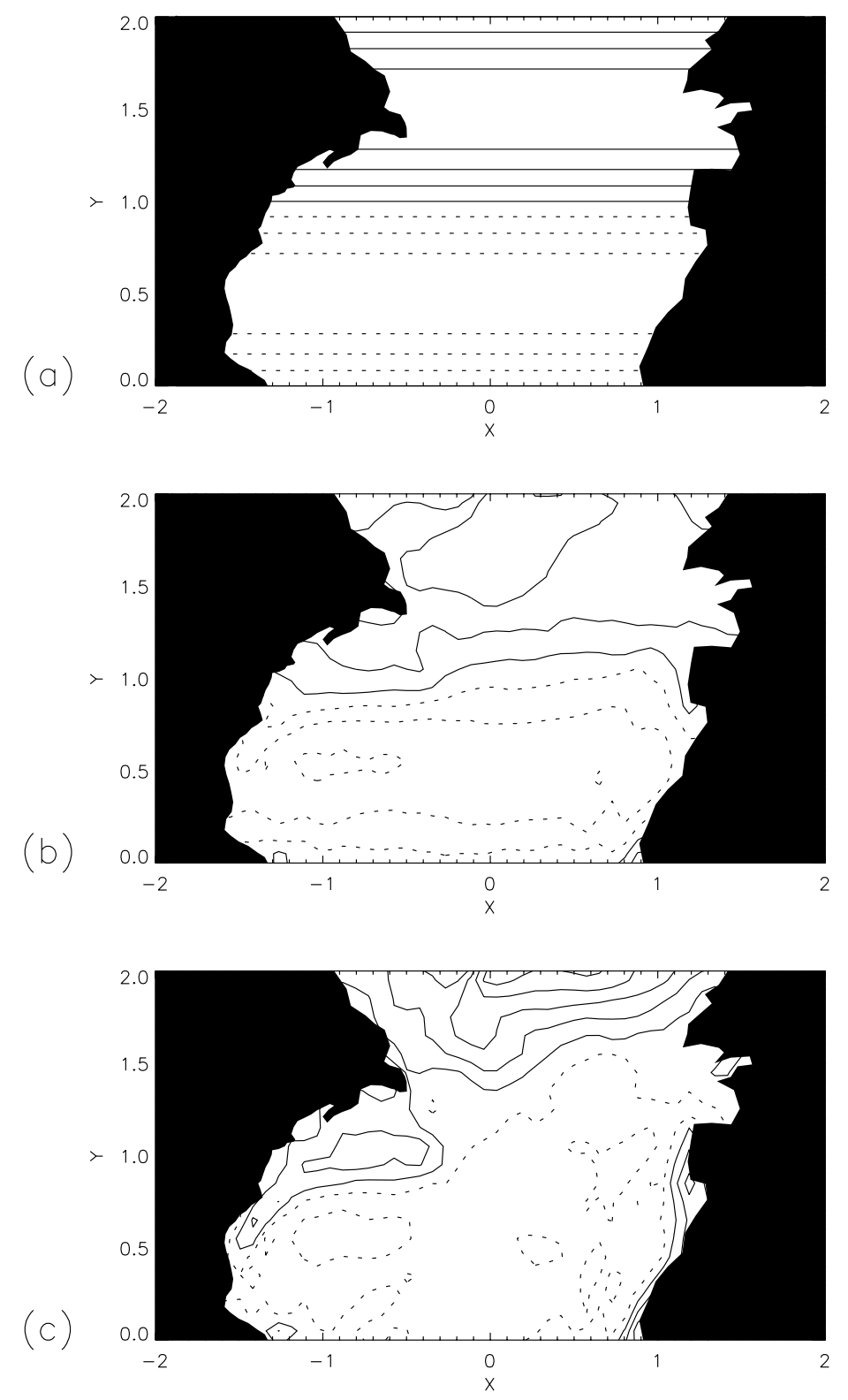

Figure 11: H.A. Dijkstra and M.J. Molemaker 


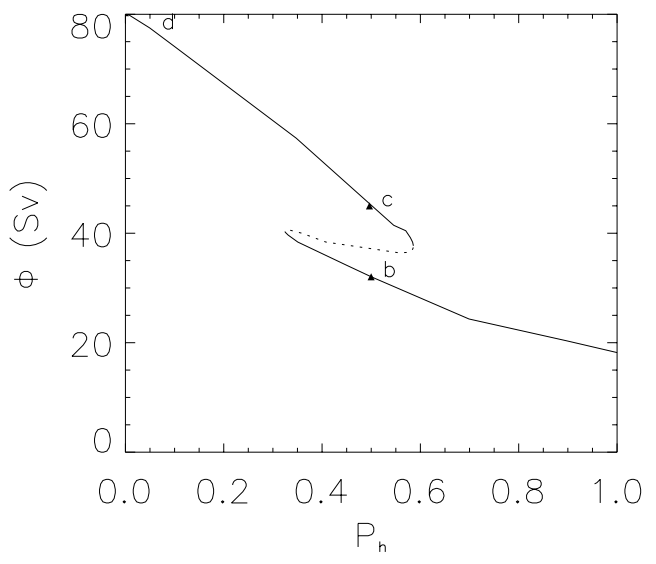

(a)

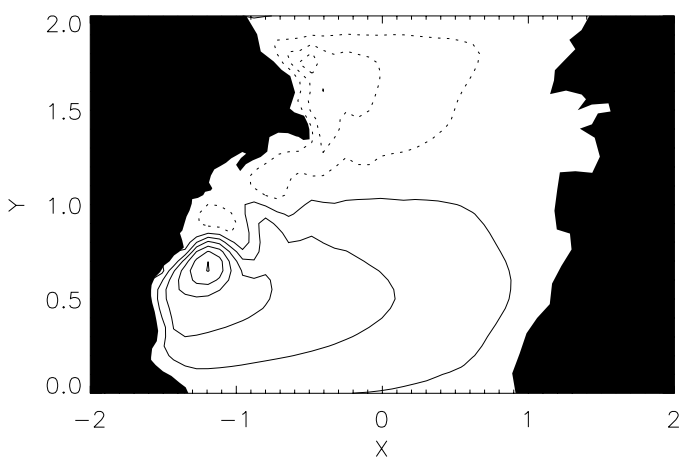

(c)

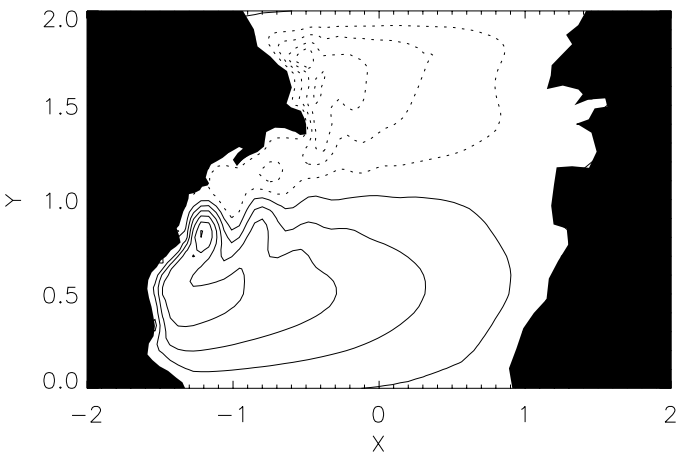

(b)

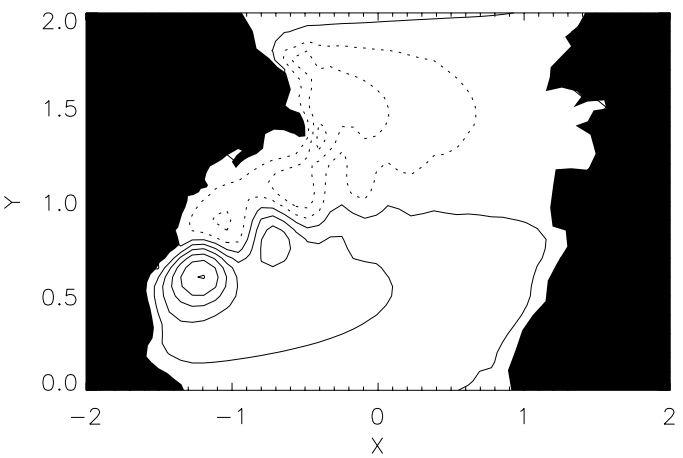

(d)

Figure 12: H.A. Dijkstra and M.J. Molemaker 


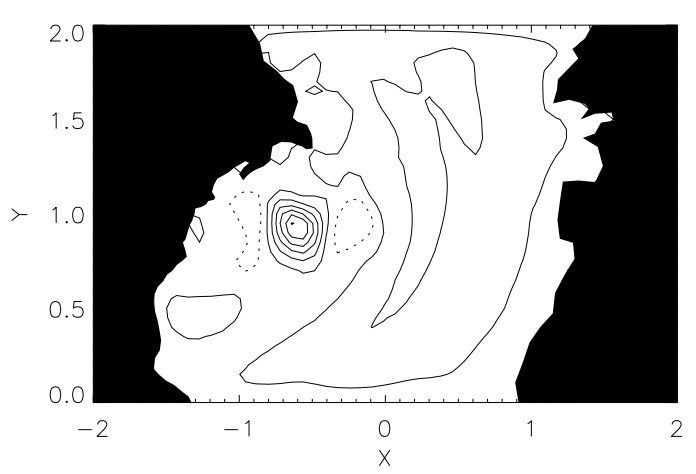

(a)

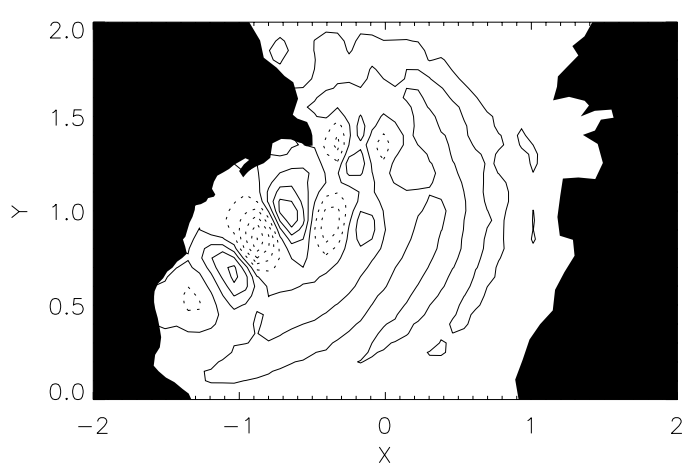

(c)

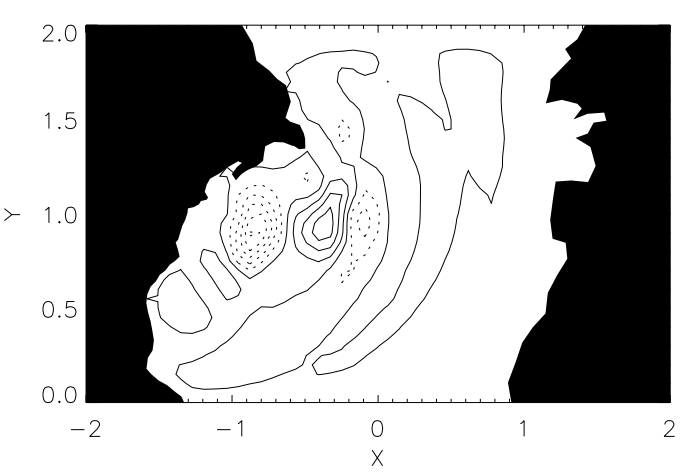

(b)

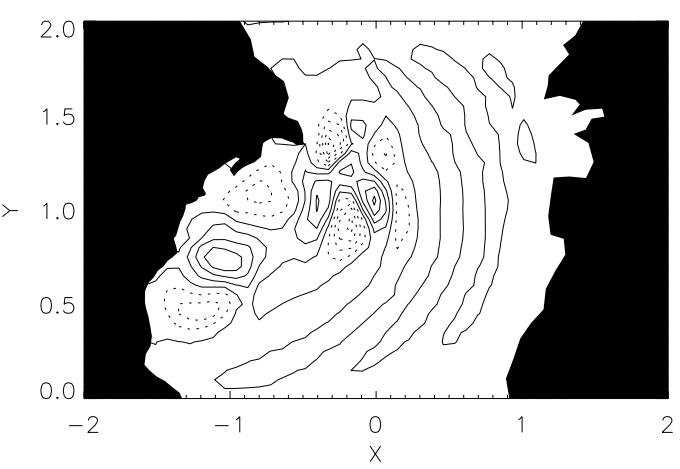

(d)

Figure 13: H.A. Dijkstra and M.J. Molemaker

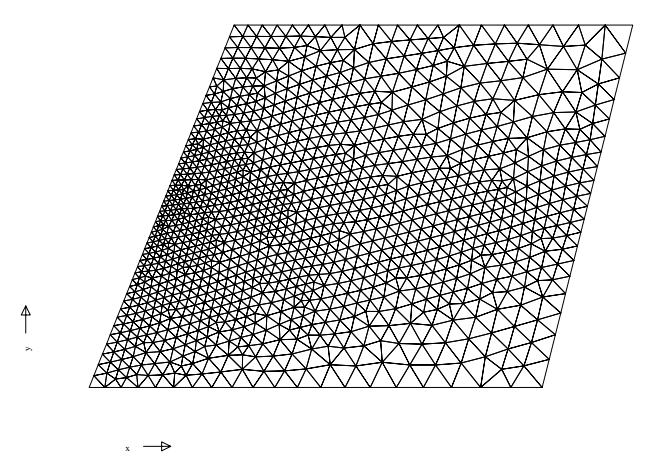

(a)

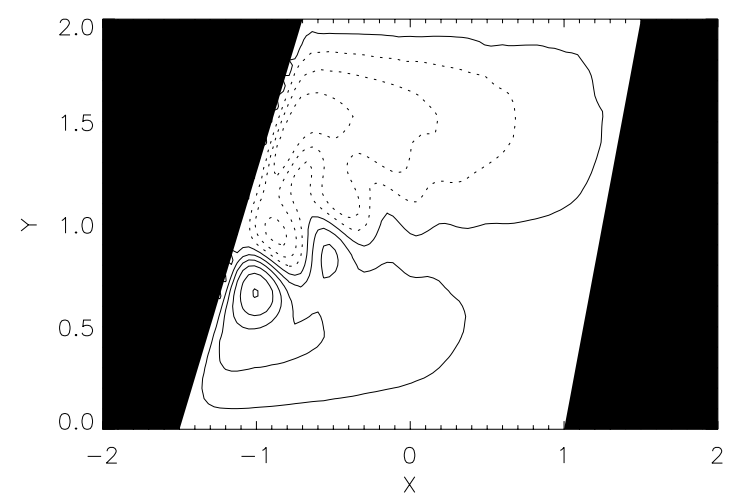

(b)

Figure 14: H.A. Dijkstra and M.J. Molemaker 


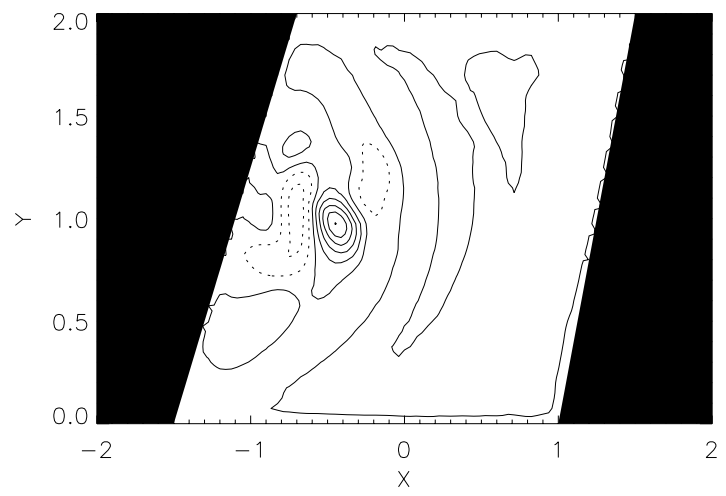

(a)

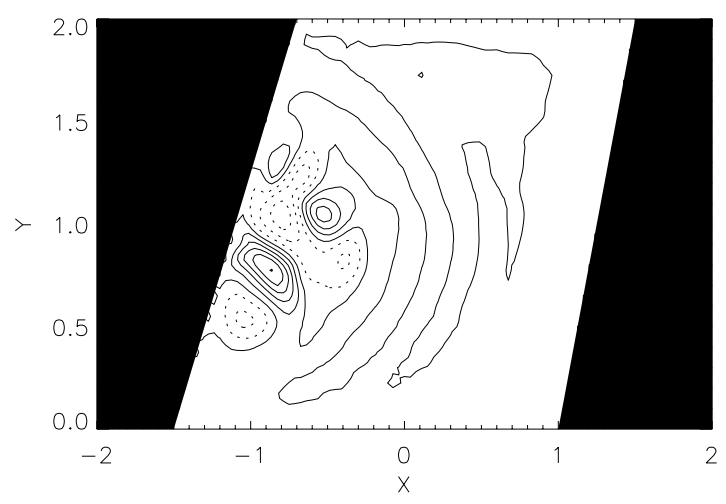

(c)

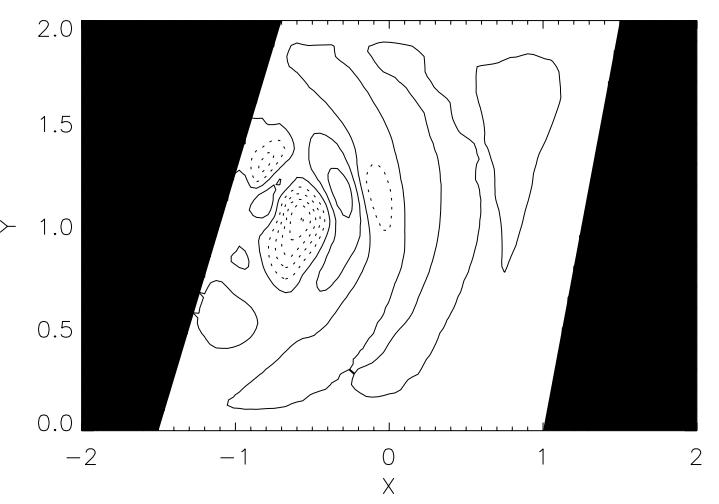

(b)

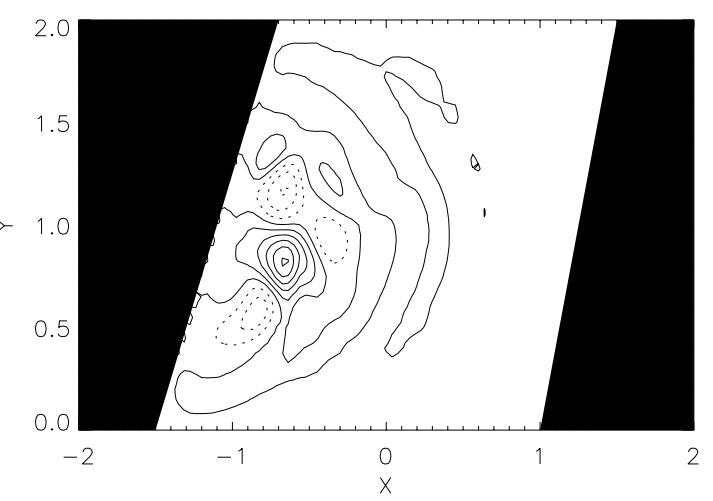

(d)

Figure 15: H.A. Dijkstra and M.J. Molemaker 